\title{
Facile Synthesis and Structure of Novel 2,5-Disubstituted 1,3,4-Selenadiazoles
}

\author{
Guoxiong Hua, ${ }^{[\mathrm{a}]}$ Yang Li, ${ }^{[\mathrm{a}]}$ Amy L. Fuller, ${ }^{[\mathrm{a}]}$ Alexandra M. Z. Slawin ${ }^{[\mathrm{a}]}$ and J. Derek Woollins ${ }^{[\mathrm{a}] *}$
}

Key words: Woollins' reagent / 1,2-diacylhydrazines / 2,5-disubstituted-1,3,4-selenadiazoles / X-ray structure

The reaction of hydrazide with carbonyl chloride in the presence of sodium carbonates lead to the corresponding 1,2-diacylhydrazines (1a-t, $\mathrm{R}^{1} \mathrm{C}(\mathrm{O}) \mathrm{NHNHC}(\mathrm{O}) \mathrm{R}^{2}, \mathrm{R}^{1}=\operatorname{aryl}, \mathrm{R}^{2}=$ aryl or alkyl) in moderate to excellent yield (57 - 90\%). The latter react with 2,4-bis(phenyl)-1,3-diselenadiphosphetane-2,4-diselenide (Woollins' reagent, WR) in refluxing toluene to give a series of new 2,5-disubstituted-1,3,4-selenadiazoles (2a-t, 51-99\% yield). All compounds were characterized spectroscopically and six compounds were characterized crystallographically.

(C) WILEY-VCH Verlag GmbH \& Co. KGaA, 69451 Weinheim, Germany, 2009)

[a] School of Chemistry, University of St Andrews, Fife, Scotland, KY16 9ST, UK

Fax: +0044 1334463384

Email: jdw3@st-and.ac.uk

\section{Introduction}

The synthesis of an organoselenium compound, diethyl selenide, was first reported in 1836. ${ }^{[1]}$ It was not until the 1970s, where the use of diethyl selenide in several new reactions created a variety of novel structures with unusual properties, that these compounds began to attract more general interest. The interest in using organoselenium heterocycles as potential pharmaceuticals and new materials, as well as reagents and catalysts expanded rapidly during last three decades. ${ }^{[2]}$ For example, 1,3,4-selenadiazoles have been studied because of their potential activities as anti-bacterial, analgesic, anti-tumor, anticonvulsant, and anti-inflammatory drugs, pesticides and fungicides. ${ }^{[3]}$ Furthermore, some of them have been used as thermotropic liquid crystals, corrosion and oxidation inhibitors, or as dyes or metal ion complexation reagents. ${ }^{[4]}$ However, little is known about the 2,5-disubstituted 1,3,4-selenadiazoles. ${ }^{[5]}$ Several methods have been reported for their preparation, which include a ring-closure reaction of selenobenzamides with hydrazine hydrate, ${ }^{[6]}$ reacting dimethylformamide azine with hydrogen selenide, ${ }^{[7]}$ treatment of 1,2 -diacetylhydrazine with phosphorus pentaselenide, ${ }^{[8]}$ reaction of isoselenocyanates with selenosemicarbazides ${ }^{[9]}$ or a carboxylic acid with selenosemicarbazide and 
phosphoryl chloride. ${ }^{[10]}$ However, the examples of these compounds in the literature are limited due to either lack of starting materials or very low yield.

2,4-Bis(phenyl)-1,3-diselenadiphosphetane 2,4-diselenide $\left[\mathrm{PhP}(\mathrm{Se})(\mu \text {-Se) }]_{2}\right.$, known as Woollins' reagent (WR), is the selenium counterpart of the well-known Lawesson's reagent $\left[p-\mathrm{MeOC}_{6} \mathrm{H}_{4} \mathrm{P}(\mathrm{S})(\mu\right.$ S) $]_{2}$ (LR). LR has been used extensively for various thionation reactions. ${ }^{[11]}$ Compared with other selenation reagents WR has broad utility and is useful in the synthesis of a wide range of seleniumcontaining compounds. These compounds include large P/Se molecular aggregates or metal complexes by nucleophilic ring-opening reactions with alkali-metal thiolates, ${ }^{[12]}$ selenoamides and selenoaldehydes by simple oxygen/selenium exchange or reaction with $\mathrm{ArCN}$ followed by hydrolysis and a wide variety of P-Se heterocycles. ${ }^{[13]}$ Here, we report the synthesis and characterization of a series of novel 2,5disubstituted 1,3,4-selenadiazoles from the selenation of the corresponding 1,2-diacylhydrazine with WR. To the best of our knowledge, this is the first report of the systematic synthesis of 2,5-disubstituted 1,3,4-selenadiazoles. Furthermore, six examples, representing the first examples of this class have been structurally characterised.

\section{Results and Discussion}

Preparation of 1,2-diacylhydrazines 1a-t was very straightforward using a modification of the literature method. ${ }^{[14]}$ Reaction between hydrazides and carbonyl chlorides in the presence of sodium carbonate leads to the corresponding 1a-t in good to excellent yields (57-90\%, Scheme 1 and Table 1). Even though most of the 1,2-diacylhydrazines had been previously synthesized; our modification of the literature preparation improved the yield and synthetic efficiency of their preparation. 1,2Diacylhydrazines, 1a-t, were characterised by IR, ${ }^{1} \mathrm{H}$ NMR, ${ }^{13} \mathrm{C}$ NMR and MS data. The Infrared spectrum of these compounds show a strong absorption in the region of $1599-1637 \mathrm{~cm}^{-1}$ and the ${ }^{13} \mathrm{C}$ NMR spectra display a characteristic peak in the region of 139.0-171.1 ppm, both are assignable to characteristic carbonyl groups, confirming the formation of 1,2-diacylhydrazines. There is also a characteristic ${ }^{1} \mathrm{H}$ NMR resonance from the NHNH group in the region of 9.80-10.69 ppm. Finally, the compounds display clear molecular ion peaks in their mass spectra. 


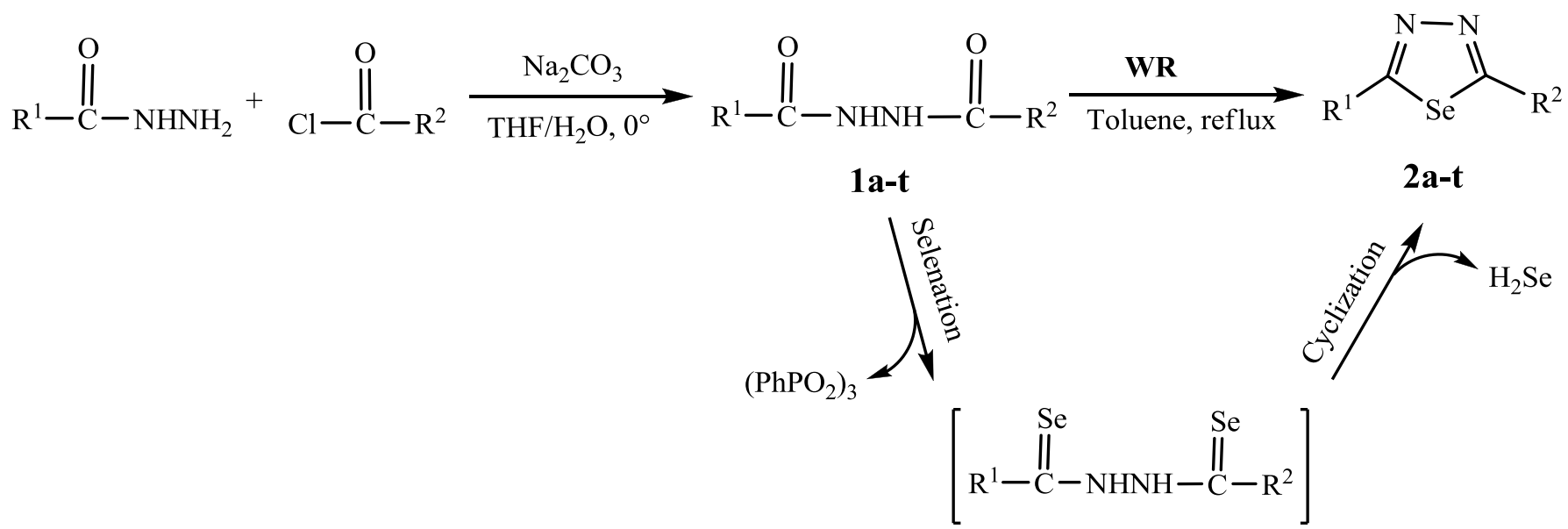

Scheme 1. Synthesis of 1,2-diacylhydrazines 1a-t and 2,5-disubstituted 1,3,4-selenadiazoles 2a-t

2,5-Disubstituted 1,3,4-thiadiazoles, the sulfur counterpart of 2,5-disubstituted 1,3,4-selenadiazoles, have been examined as potential anti-bacterial, ${ }^{[15]}$ anti-viral, ${ }^{[16]}$ analgesic, ${ }^{[17]}$ anti-tumor, ${ }^{[18]}$ anticonvulsant, ${ }^{[15]}$ and anti-inflammatory drugs, along with activity as pesticides and fungicides, ${ }^{[17,19]}$ as well as other applications. ${ }^{[4 a-c, 20]}$ The most popular method for the synthesis of this class of compounds involves the cyclization and dehydration of thiohydrazides or other substrates with S-C-N-N-C-S moiety. ${ }^{[4 a, 15,16]}$ This is typically done via thionation of 1,2-diacylhydrazines with Lawesson's reagent, followed by spontaneous cyclization and dehydrosulfurization. ${ }^{[14 c]}$ Herein, we adopted a similar approach, that is, reacting 1,2-diacylhydrazines, 1a-t, with WR to afford a series of 2,5-disubstituted 1,3,4-selenadiazoles, 2a-t, in moderate to excellent yield (51-99\%, Scheme 1 and Table 1). It should be noted in particular that reaction of $\mathbf{W R}$ with 1,2-diacylhydrazines bearing diaryl groups $\left(\mathrm{R}^{1}, \mathrm{R}^{2}=\right.$ Aryl $)$ is very fast and high yield (75-99\%), while with the 1,2-diacylhydrazines bearing one aryl group and one pyridine group $\left(\mathrm{R}^{1}=\right.$ Aryl; $\mathrm{R}^{2}=\mathrm{Pyr}$ ) is slow with relatively low yield (51 and $58 \%$ for compounds $\mathbf{2 b}$ and 2c, respectively), This is most likely due to the presence of the electron-withdrawing pyridine group. $\mathbf{2}$ are stable in air and moisture for several months and are soluble in common organic solvents. 
Table 1. Synthesis of 1,2-diacylhydrazines 1a-t and 2,5-disubstituted 1,3,4-selenadiazoles 2a-t

\begin{tabular}{|c|c|c|c|c|c|}
\hline $\mathrm{R}^{1}$ & $\mathrm{R}^{2}$ & 1a-t & Yield (\%) & $2 \mathbf{a}-\mathbf{t}$ & Yield (\%) \\
\hline $\mathrm{C}_{6} \mathrm{H}_{5}$ & $\mathrm{C}_{6} \mathrm{H}_{5}$ & $1 \mathbf{a}$ & 61 & $2 \mathbf{a}$ & 98 \\
\hline $4-\mathrm{BrC}_{6} \mathrm{H}_{4}$ & $4-\mathrm{BrC}_{6} \mathrm{H}_{4}$ & $1 b$ & 65 & $2 b$ & 75 \\
\hline $\mathrm{C}_{6} \mathrm{H}_{5}$ & Pyridin-3-yl & 1c & 77 & $2 c$ & 51 \\
\hline $4-\mathrm{MeOC}_{6} \mathrm{H}_{4}$ & Pyridin-3-yl & 1d & 82 & $2 d$ & 58 \\
\hline $\mathrm{C}_{6} \mathrm{H}_{5}$ & 4- $\mathrm{MeC}_{6} \mathrm{H}_{4}$ & 1e & 63 & $2 e$ & 90 \\
\hline $\mathrm{C}_{6} \mathrm{H}_{5}$ & 4- $\mathrm{ClC}_{6} \mathrm{H}_{4}$ & 1f & 67 & $2 f$ & 97 \\
\hline $\mathrm{C}_{6} \mathrm{H}_{5}$ & 4- $\mathrm{BrC}_{6} \mathrm{H}_{4}$ & $1 g$ & 69 & $2 \mathrm{~g}$ & 95 \\
\hline $\mathrm{C}_{6} \mathrm{H}_{5}$ & $4-\mathrm{FC}_{6} \mathrm{H}_{4}$ & $1 \mathrm{~h}$ & 81 & $2 \mathrm{~h}$ & 91 \\
\hline $\mathrm{C}_{6} \mathrm{H}_{5}$ & Thiophen-2-yl & $1 \mathbf{i}$ & 70 & $2 \mathbf{i}$ & 97 \\
\hline $\mathrm{C}_{6} \mathrm{H}_{5}$ & $\mathrm{C}_{2} \mathrm{H}_{5} \mathrm{O}$ & $\mathbf{1 j}$ & 90 & $2 \mathbf{j}$ & 99 \\
\hline $\mathrm{C}_{6} \mathrm{H}_{5}$ & Furan-2-yl & $1 \mathbf{k}$ & 81 & $2 \mathbf{k}$ & 82 \\
\hline 4- $\mathrm{MeC}_{6} \mathrm{H}_{4}$ & Furan-2-yl & 11 & 65 & 21 & 90 \\
\hline $4-\mathrm{BrC}_{6} \mathrm{H}_{4}$ & Furan-2-yl & $1 \mathbf{m}$ & 63 & $2 \mathrm{~m}$ & 96 \\
\hline $4-\mathrm{MeOC}_{6} \mathrm{H}_{4}$ & 4- $\mathrm{MeC}_{6} \mathrm{H}_{4}$ & 1n & 69 & $2 n$ & 90 \\
\hline $4-\mathrm{MeOC}_{6} \mathrm{H}_{4}$ & $\mathrm{C}_{6} \mathrm{H}_{5}$ & 1p & 77 & $2 p$ & 86 \\
\hline $4-\mathrm{MeC}_{6} \mathrm{H}_{4}$ & $4-\mathrm{ClC}_{6} \mathrm{H}_{4}$ & $1 \mathbf{r}$ & 68 & $2 r$ & 83 \\
\hline $4-\mathrm{MeC}_{6} \mathrm{H}_{4}$ & $4-\mathrm{FC}_{6} \mathrm{H}_{4}$ & $1 \mathrm{~s}$ & 57 & $2 s$ & 79 \\
\hline $4-\mathrm{MeC}_{6} \mathrm{H}_{4}$ & $4-\mathrm{BrC}_{6} \mathrm{H}_{4}$ & $1 t$ & 81 & $2 t$ & 75 \\
\hline
\end{tabular}

The characterisation of $\mathbf{2 a - t}$ is based on elemental analyses, ${ }^{1} \mathrm{H},{ }^{13} \mathrm{C}$ and ${ }^{77} \mathrm{Se} \mathrm{NMR}$, IR spectroscopy and mass spectrometry. The elemental analyses for all of these new compounds were satisfactory. All of compounds showed the anticipated molecular ion peaks $[\mathrm{M}]^{+},[\mathrm{M}-\mathrm{H}]^{+},[\mathrm{M}+\mathrm{H}]^{+}$or $[\mathrm{M}+\mathrm{Na}]^{+}$in their mass spectra. The $v(\mathrm{C}=\mathrm{N})$ vibrations are observed in the range of $1418-1495 \mathrm{~cm}^{-1}$, comparable with related hetercycles. ${ }^{[1]}$ The absence of $v\left(1599-1637 \mathrm{~cm}^{-1}\right.$ for $\left.\mathrm{C}=\mathrm{O}\right)$ and the presence of the typical ${ }^{13} \mathrm{C}$ NMR signals in the range of 133.1-174.9 ppm for $\mathrm{C}=\mathrm{N}$ double bond and ${ }^{77} \mathrm{Se}$ NMR signals in the range of 683.01-714.61 ppm indicate the formation of the five-membered ring systems, 2a-t.

Surprisingly, to date, there have been no crystal structures of 1,3,4-selenadiazole yet reported. Here we report the structures of six examples $\mathbf{2 b}, \mathbf{2 h}, \mathbf{2 k}, \mathbf{2 m}, \mathbf{2 \mathbf { p }}$ and $\mathbf{2 s}$ (Figure 1). Colourless block crystals of these compounds were obtained for X-ray analysis via slow evaporation of a dichloromethane solution into hexane. Details of the selected inter-atomic distances and angles are given in Table 2. Unfortunately, the data for $\mathbf{2 g}$ could not be well-refined though the structure is closely similar to the other six described here. ${ }^{[22]}$ The overall molecular structures of these compounds are very similar in geometry. The $\mathrm{C}_{2} \mathrm{~N}_{2} \mathrm{Se}$ rings are approximately co-planar with the two aromatic substituents. This can be readily illustrated 
through a comparison of the torsion angles to the substituents which are all close to $180^{\circ}$ [range 176.1(7)-180.0(8) ${ }^{\circ}$. The C-Se bond lengths for all six compounds (ca. 1.87-1.89 ^) are similar to that of 2,5-diarylselenophenes (ca. 1.86-1.89 ̊). ${ }^{[23]}$ The distances in 2 are slightly shorter than those found in related structures containing single C-Se bonds ( $c a$. 1.92-1.94 $\AA$ ), ${ }^{[24]}$ indicating that some slight delocalisation in $\mathbf{2}$. However, the $\mathrm{C}-\mathrm{Se}-\mathrm{C}$ angles ranging between $81.9(2)-82.7(2)^{\circ}$ in $\mathbf{2 b}, \mathbf{2 h}, \mathbf{2 k}, \mathbf{2 m}, \mathbf{2 p}$ and $2 \mathrm{~s}$ are considerably smaller than that $\left(\mathrm{ca} .87 .7(7)-88.7(10)^{\circ}\right)$ in 2,5-diarylselenophenes. ${ }^{[24]}$

(A)

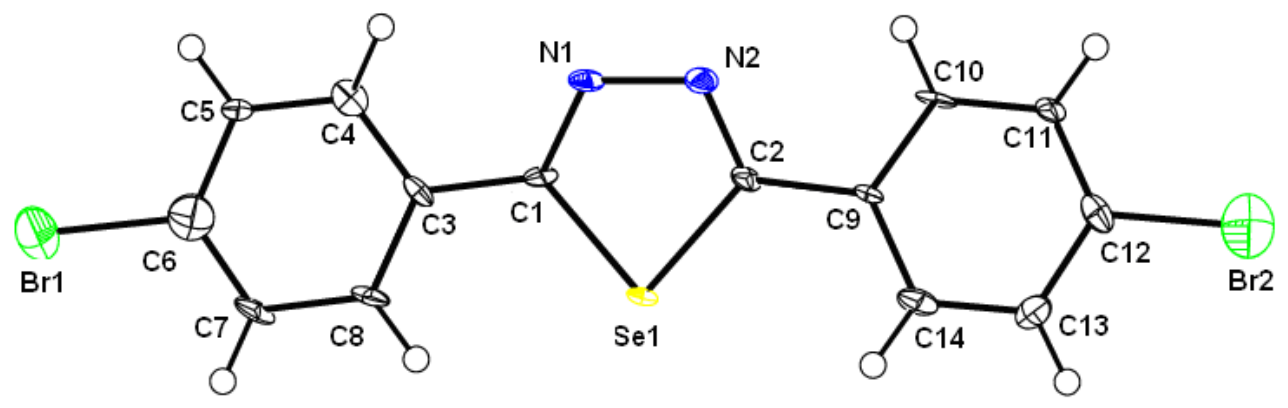

(B)



(C)

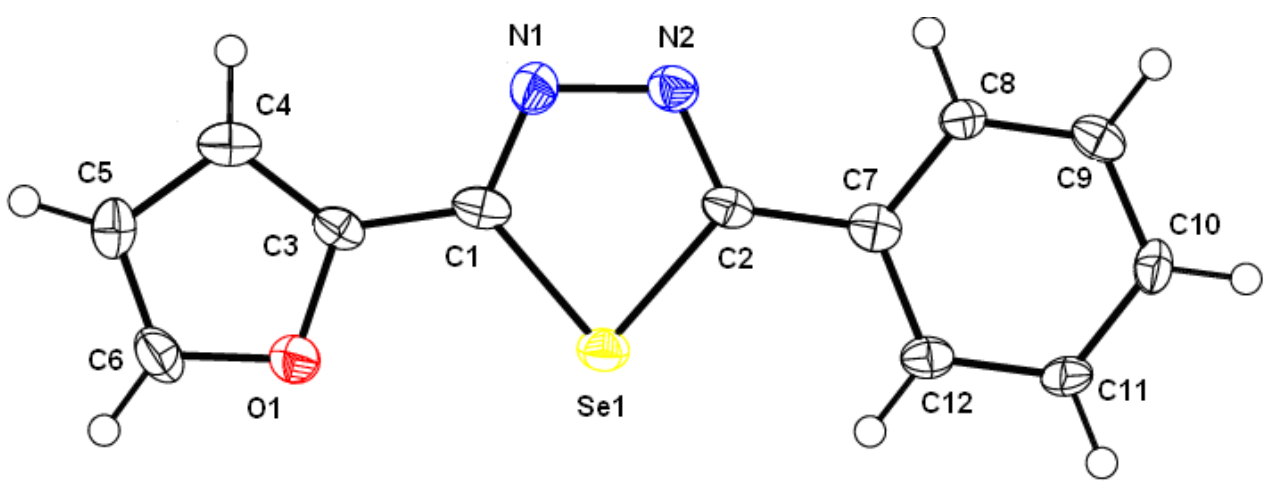


(D)

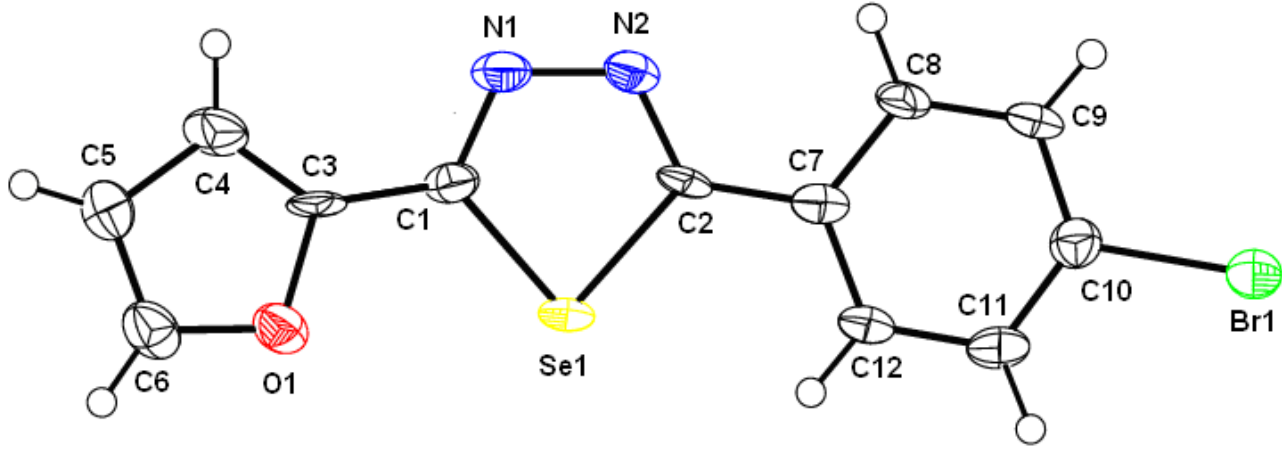

(E)

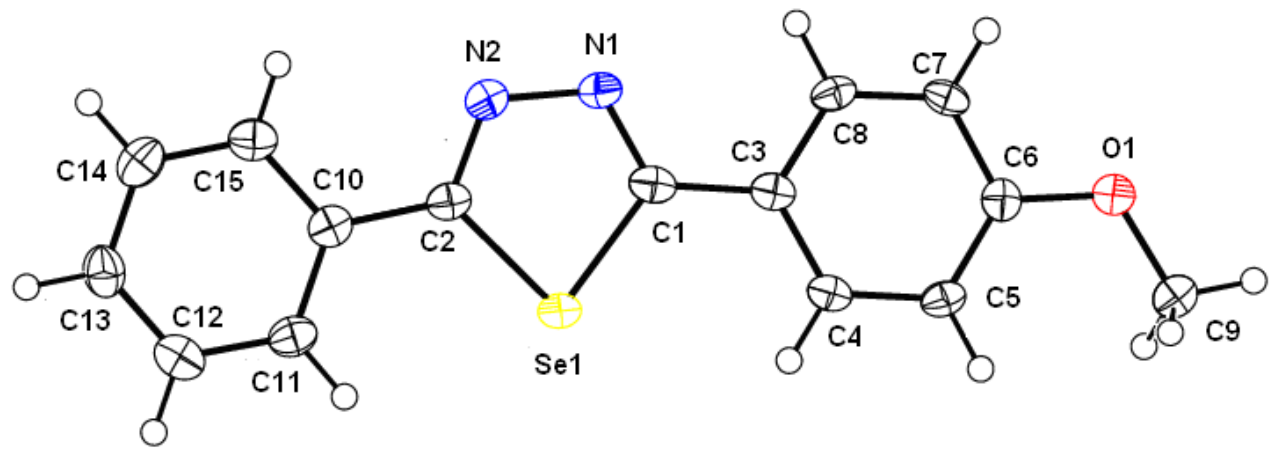

(F)



Figure 1. X-ray crystal structures of $\mathbf{2 b}(\mathrm{A}), \mathbf{2 h}(\mathrm{B}), \mathbf{2 k}(\mathrm{C}), \mathbf{2 m}(\mathrm{D}), \mathbf{2 p}(\mathrm{E})$ and $\mathbf{2 s}(\mathrm{F})$ 
Table 2. Selected interatomic distances $(\AA \hat{)})$ and angles $\left({ }^{\circ}\right)$ for $\mathbf{2 b}, \mathbf{2 h}, \mathbf{2 k}, \mathbf{2 m}, \mathbf{2 p}$ and $\mathbf{2 s}$

\begin{tabular}{lcccccc}
\hline & $\mathbf{2 b}$ & $\mathbf{2 h}$ & $\mathbf{2 k}$ & $\mathbf{2 m}$ & $\mathbf{2 p}$ & $\mathbf{2 s}$ \\
\hline $\mathrm{Se}(1)-\mathrm{C}(1)$ & $1.885(10)$ & $1.891(7)$ & $1.861(5)$ & $1.869(9)$ & $1.880(3)$ & $1.902(5)$ \\
$\mathrm{Se}(1)-\mathrm{C}(2)$ & $1.884(10)$ & $1.884(7)$ & $1.887(5)$ & $1.894(8)$ & $1.877(3)$ & $1.890(5)$ \\
$\mathrm{C}(2)-\mathrm{N}(2)$ & $1.295(13)$ & $1.296(9)$ & $1.302(7)$ & $1.299(12)$ & $1.298(4)$ & $1.301(6)$ \\
$\mathrm{N}(2)-\mathrm{N}(1)$ & $1.381(12)$ & $1.363(8)$ & $1.374(7)$ & $1.380(10)$ & $1.373(4)$ & $1.379(6)$ \\
$\mathrm{C}(1)-\mathrm{N}(1)$ & $1.276(12)$ & $1.307(9)$ & $1.302(7)$ & $1.295(12)$ & $1.297(4)$ & $1.294(6)$ \\
& & & & & & \\
$\mathrm{C}(1)-\mathrm{Se}(1)-\mathrm{C}(2)$ & $81.9(4)$ & $82.0(3)$ & $81.9(2)$ & $82.2(4)$ & $82.22(15)$ & $82.7(2)$ \\
$\mathrm{Se}(1)-\mathrm{C}(2)-\mathrm{N}(2)$ & $113.9(7)$ & $114.7(5)$ & $113.7(4)$ & $112.9(6)$ & $113.8(3)$ & $113.5(4)$ \\
$\mathrm{C}(2)-\mathrm{N}(2)-\mathrm{N}(1)$ & $114.5(8)$ & $114.1(6)$ & $115.1(4)$ & $115.8(8)$ & $115.1(3)$ & $114.7(4)$ \\
$\mathrm{N}(2)-\mathrm{N}(1)-\mathrm{C}(1)$ & $115.6(8)$ & $116.5(6)$ & $114.1(5)$ & $114.0(8)$ & $115.0(3)$ & $116.8(4)$ \\
$\mathrm{N}(1)-\mathrm{C}(1)-\mathrm{Se}(1)$ & $114.0(8)$ & $112.6(5)$ & $115.2(4)$ & $115.0(7)$ & $113.9(3)$ & $112.2(4)$
\end{tabular}

\section{Conclusions}

In summary, a highly efficient route for the preparation of a wide variety of 1,2-diacylhydrazines has been developed. The selenation of 1,2-diacylhydrazines using Woollins' reagent, 2,4-bis(phenyl)-1,3diselenadiphosphetane-2,4-diselenide, provides a general and systemic approach to 2,5-disubstituted 1,3,4-selenadiazoles. This method allows 2,5-disubstituted 1,3,4-selenadiazoles to be easily available for further investigations into their chemistry and biological properties.

\section{Experimental Section}

Unless otherwise stated, all reactions were carried out under on oxygen free nitrogen atmosphere using pre-dried solvents and standard Schlenk techniques, subsequent chromatographic and work up procedures were performed in air. Solvents were dried, purified, and stored according to common procedures.

${ }^{1} \mathrm{H}(270 \mathrm{MHz}),{ }^{13} \mathrm{C}(67.9 \mathrm{MHz}),{ }^{31} \mathrm{P}-\left\{{ }^{1} \mathrm{H}\right\}(109 \mathrm{MHz})$ and ${ }^{77} \mathrm{Se}-\left\{{ }^{1} \mathrm{H}\right\}(51.4 \mathrm{MHz}$ referenced to external $\mathrm{Me}_{2} \mathrm{Se}$ ) NMR spectra were recorded at $25{ }^{\circ} \mathrm{C}$ (unless stated otherwise) on a JEOL GSX 270. IR spectra were recorded as $\mathrm{KBr}$ pellets in the range of $4000-250 \mathrm{~cm}^{-1}$ on a Perkin-Elmer 2000 FTIR/Raman spectrometer. Microanalysis was performed by the University of St-Andrews microanalysis service. Mass spectrometry was performed by the University of St Andrews Mass Spectrometry Service. X-ray crystal data as Table 3 for compounds $\mathbf{2 b}, \mathbf{2 g}, \mathbf{2 h}, \mathbf{2 k}, \mathbf{2 m}, \mathbf{2 p}$ and $2 \mathbf{s}$ was collected at $93 \mathrm{~K}$ by using a Rigaku MM007 High brilliance RA generator/confocal optics and Mercury CCD system. Intensities were corrected for Lorentz-polarisation and for absorption. The structures were solved by direct methods. Hydrogen atoms bound to carbon were idealised. Structural refinements were obtained with 
full-matrix least-squares based on $F^{2}$ by using the SHELXTL CDC-contain the supplementary crystallographic data for this paper. These data for compounds $\mathbf{2 b}, \mathbf{2 h}, \mathbf{2 k}, \mathbf{2 m}, \mathbf{2} \mathbf{p}$ and $2 \mathbf{s}$ can be obtained free of charge via www.ccdc.cam.ac.uk/conts/retrieving.html CCDC 711573-711578 or from the Cambridge Crystallographic Data centre, 12 Union Road, Cambridge CB2 1EZ, UK; Fax (+44) 1223-336-033; E-mail: deposit@ccdc.cam.ac.uk.

Table 3. Details of the X-ray data collections and refinements for $\mathbf{2 b}, \mathbf{2 h}, \mathbf{2 k}, \mathbf{2 m}, \mathbf{2 p}$ and $\mathbf{2 s}$

\begin{tabular}{|c|c|c|c|c|c|c|}
\hline & $2 \mathbf{b}$ & $2 \mathrm{~h}$ & $2 \mathbf{k}$ & $2 \mathrm{~m}$ & $2 p$ & $2 s$ \\
\hline Formula & $\mathrm{C}_{14} \mathrm{H}_{8} \mathrm{Br}_{2} \mathrm{~N}_{2} \mathrm{Se}$ & $\mathrm{C}_{14} \mathrm{H}_{9} \mathrm{FN}_{2} \mathrm{Se}$ & $\mathrm{C}_{12} \mathrm{H}_{8} \mathrm{~N}_{2} \mathrm{OSe}$ & $\mathrm{C}_{12} \mathrm{H}_{7} \mathrm{BrN}_{2} \mathrm{OSe}$ & $\mathrm{C}_{15} \mathrm{H}_{12} \mathrm{~N}_{2} \mathrm{OSe}$ & $\mathrm{C}_{15} \mathrm{H}_{11} \mathrm{FN}_{2} \mathrm{Se}$ \\
\hline$M$ & 443.00 & 303.19 & 275.16 & 354.07 & 315.23 & 317.22 \\
\hline Crystal system & Monoclinic & Monoclinic & Orthorhombic & Monoclinic & Orthorhombic & Monoclinic \\
\hline Space group & $P 2_{1} / \mathrm{c}$ & $P 2_{1} / \mathrm{c}$ & $P 2_{1} 2_{1} 2_{1}$ & $P 2_{1} / \mathrm{c}$ & Pbca & $P 2_{1} / \mathrm{c}$ \\
\hline$a / \AA ̊$ & $15.421(6)$ & $13.160(6)$ & $7.217(3)$ & $6.112(3)$ & $14.222(6)$ & $14.888(8)$ \\
\hline$b / \AA$ & $14.353(5)$ & 5.489(3) & $13.857(5)$ & $21.886(9)$ & $6.056(3)$ & $14.385(7)$ \\
\hline$c / \AA$ & $6.121(2)$ & $16.177(8)$ & $10.474(3)$ & $8.871(4)$ & $29.774(15)$ & $6.002(3)$ \\
\hline$\beta$ & 101.091(10) & $99.108(12)$ & 90 & $103.712(10)$ & 90 & $97.699(13)$ \\
\hline$U / A^{3}$ & $1329.4(8)$ & $1153.8(10)$ & $1047.4(6)$ & $1152.8(9)$ & $2564(2)$ & $1273.9(11)$ \\
\hline$Z$ & 4 & 4 & 4 & 4 & 8 & 4 \\
\hline$\mu / \mathrm{mm}^{-1}$ & 8.82 & 3.25 & 3.56 & 6.71 & 2.92 & 2.95 \\
\hline $\begin{array}{l}\text { Reflections } \\
\text { collected } \\
\text { Independent }\end{array}$ & 8630 & 7127 & 7030 & 7733 & 15423 & 8122 \\
\hline reflections & 2678 & 2266 & 2152 & 2277 & 2582 & 2610 \\
\hline$R_{\text {int }}$ & 0.120 & 0.103 & 0.101 & 0.217 & 0.049 & 0.161 \\
\hline $\begin{array}{l}R \quad\left[\mathrm{~F}^{2}>\right. \\
\left.2 \sigma\left(\mathrm{F}^{2}\right)\right]\end{array}$ & 0.094 & 0.075 & 0.048 & 0.098 & 0.045 & 0.086 \\
\hline $\begin{array}{ll}\mathrm{w} R 2 & \quad \mathrm{I}> \\
2 \sigma(\mathrm{I})] & \\
\end{array}$ & 0.273 & 0.252 & 0.114 & 0.308 & 0.088 & 0.227 \\
\hline
\end{tabular}

General procedure for synthesis of 1,2-diacylhydrazines (1a-t) A suspension of carbonyl hydrazide (10.0 mmol) and anhydrous sodium carbonate $(1.02 \mathrm{~g}, 10.0 \mathrm{mmol})$ in dry tetrahydrofuran $(60 \mathrm{ml})$ and water $(60 \mathrm{ml})$ was added to a stirring solution of the corresponding carbonyl chloride (11.0 mmol) in 30 $\mathrm{ml}$ of tetrahydrofuran at $0^{\circ} \mathrm{C}$. The mixture was stirred at $0^{\circ} \mathrm{C}$ for $1 \mathrm{~h}$, and at room temperature for $4 \mathrm{~h}$. A massive precipitation was observed. The product was harvested by filtration and washed three times with tetrahydrofuran and ethyl ether, then finally dried in vacuum.

$N^{\prime}$-Benzoylbenzohydrazide (1a). A white solid $(61 \%, 1.45 \mathrm{~g})$; m.p. $236-238^{\circ} \mathrm{C}$. Selected IR (KBr, $\left.\mathrm{cm}^{-1}\right)$ : 3201(s), 3001(s), 1670(m), 1633(vs), 1579(s), 1537(s), 1487(s), 1287(s), 687(m). ${ }^{1} \mathrm{H}$ NMR (DMSO-d 6 , 


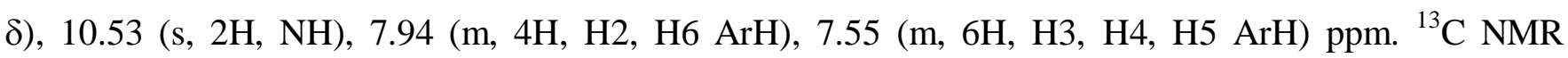
$\left(\mathrm{DMSO}_{\mathrm{d}}, \delta\right), 166.3(\mathrm{C}=\mathrm{O}), 133.3,132.4,129.1,128.0$ ppm. MS (CI, m/z), $241[\mathrm{M}+\mathrm{H}]^{+}$.

4-Bromo- $N^{\prime}$-(4-chlorobenzoyl)benzohydrazide (1b). A white solid (65\%, 2.30 g); m.p. 302-303 C. Selected IR (KBr, cm $\left.{ }^{-1}\right)$ : 3187(s), 3017(w), 1601(vs), 1561(s), 1461(s), 1264(m), 1090(m), 1010(m), 848(m), 742(m), 659(m). ${ }^{1} \mathrm{H}$ NMR $\left(\mathrm{DMSO}_{6}, \delta\right), 10.66(\mathrm{~s}, 2 \mathrm{H}, \mathrm{NH}), 7.95(\mathrm{~d}, J(\mathrm{H}, \mathrm{H})=8.5 \mathrm{~Hz}, 2 \mathrm{H}$, $\operatorname{ArH}), 7.87(\mathrm{~d}, J(\mathrm{H}, \mathrm{H})=8.5 \mathrm{~Hz}, 2 \mathrm{H}, \operatorname{ArH}), 7.75(\mathrm{~d}, J(\mathrm{H}, \mathrm{H})=8.5 \mathrm{~Hz}, 2 \mathrm{H}, \mathrm{ArH}), 7.62(\mathrm{~d}, J(\mathrm{H}, \mathrm{H})=8.5 \mathrm{~Hz}$, $2 \mathrm{H}, \mathrm{ArH}) \mathrm{ppm} .{ }^{13} \mathrm{C}$ NMR (DMSO-d 6 , $\left.\delta\right), 165.5(\mathrm{C}=\mathrm{O}), 165.4(\mathrm{C}=\mathrm{O}), 137.4,132.2,132.1,131.8,130.1$, 130.0, 129.3, 126.3 ppm. MS $\left.\left(\mathrm{ES}^{+}, \mathrm{m} / \mathrm{z}\right), 377[\mathrm{M}+\mathrm{Na}]^{+} . \mathrm{MS}_{(\mathrm{ES}}, \mathrm{m} / \mathrm{z}\right), 353[\mathrm{M}-\mathrm{H}]^{+}$.

$N^{\prime}$-Benzoylnicotinohydrazide (1c). A white solid (77\%, 1.85 g); m.p. 232-234 ${ }^{\circ}$. Selected IR (KBr, $\left.\mathrm{cm}^{-1}\right)$ : 3201(m), 3003(m), 1631(s), 1537(s), 1295(s), 874(m), 698(s). ${ }^{1} \mathrm{H}$ NMR (DMSO-d 6 , $), 10.69$ (s, 2H, NH), 9.09 (s, 1H, PyH), 8.80 (d, 1H, PyH), 8.29 (d, 1H, PyH), 7.94 (m, 2H, ArH), 7.57 (m, 4H, PyH and $\mathrm{ArH}) \mathrm{ppm} .{ }^{13} \mathrm{C}$ NMR (DMSO-d $\left.6, \delta\right), 166.3(\mathrm{C}=\mathrm{O}), 165.0(\mathrm{C}=\mathrm{O}), 153.1,149.0,135.8,133.0,132.5$, 129.1, 128.9, 128.0, 124.3 ppm. MS (CI, m/z), $242[\mathrm{M}+\mathrm{H}]^{+}$.

$N^{\prime}$-(4-Methoxybenzoyl)nicotinohydrazide (1d). A white solid $(82 \%, 2.21 \mathrm{~g})$; m.p. 126-128 ${ }^{\circ} \mathrm{C}$. Selected IR (KBr, cm $\left.{ }^{-1}\right)$ : 3203(m), 3003(m), 1632(s), 1605(s), 1503(s), 1258(s), 1175(m), 1026(m), 846(w), 704(w). ${ }^{1} \mathrm{H}$ NMR (DMSO-d 6 , $), 10.66$ (s, 2H, NH), 9.10 (s, 1H, PyH), 8.79 (d, 1H, PyH), 8.30 (d, 1H, $\mathrm{PyH}), 7.93$ (m, 2H, ArH), 7.58 (m, 1H, PyH), 7.07 (m, 2H, ArH), 3.84 (s, 3H, OCH $)$ ppm. ${ }^{13} \mathrm{C}$ NMR $\left(\mathrm{DMSO}_{-} \mathrm{d}_{6}, \delta\right), 165.9(\mathrm{C}=\mathrm{O}), 165.1(\mathrm{C}=\mathrm{O}), 153.1,149.0,135.8,129.9,128.9,125.1,124.3,114.4,114.0$, $56.0 \mathrm{ppm}$. MS (CI, m/z), $272[\mathrm{M}+\mathrm{H}]^{+}$.

N'-Benzoyl-4-methylbenzohydrazide (1e). A white solid (63\%, 1.58 g); m.p. 219-221 ${ }^{\circ}$ C. Selected IR $\left(\mathrm{KBr}, \mathrm{cm}^{-1}\right)$ : 3202(s), 3007(s), 1631(vs), 1578(m), 1539(s), 1489(m), 1284(s), 691(m). ${ }^{1} \mathrm{H}$ NMR $\left(\mathrm{DMSO}_{\mathrm{d}}, \delta\right), 10.45(\mathrm{~s}, 2 \mathrm{H}, \mathrm{NH}), 7.93(\mathrm{~d}, J(\mathrm{H}, \mathrm{H})=7.7 \mathrm{~Hz}, 2 \mathrm{H}, \mathrm{ArH}), 7.84(\mathrm{~d}, J(\mathrm{H}, \mathrm{H})=7.4 \mathrm{~Hz}, 2 \mathrm{H}$, ArH), $7.55(\mathrm{~m}, 3 \mathrm{H}, \mathrm{ArH}), 7.32(\mathrm{~d}, J(\mathrm{H}, \mathrm{H})=7.4 \mathrm{~Hz}, 2 \mathrm{H}, \mathrm{ArH}), 2.38\left(\mathrm{~s}, 3 \mathrm{H}, \mathrm{CH}_{3}\right)$ ppm. ${ }^{13} \mathrm{C} \mathrm{NMR}$ $\left(\mathrm{DMSO}_{\mathrm{d}}, \delta\right), 166.4(\mathrm{C}=\mathrm{O}), 166.2(\mathrm{C}=\mathrm{O}), 142.4,133.3,132.4,130.4,129.6,129.1,128.0,21.6 \mathrm{ppm}$. MS (CI, m/z), $255[\mathrm{M}+\mathrm{H}]^{+}$.

$N^{\prime}$-Benzoyl-4-chlorobenzohydrazide (1f). A white solid $(67 \%, 1.96$ g); m.p. 224-226 C. Selected IR $\left(\mathrm{KBr}, \mathrm{cm}^{-1}\right):$ 3195(s), 3010(w), 2843(w), 1599(vs), 1564(s), 1498(m), 1461(s), 1266(m), 1089(m), 1010(m), 849(m), 710(m), 687(m), 647(m), 454(m). ${ }^{1} \mathrm{H}$ NMR (DMSO-d $\left.6, \delta\right), 10.60$ (s, 2H, NH), 7.94 $(\mathrm{m}, 5 \mathrm{H}, \mathrm{ArH}), 7.55$ (m, 4H, ArH) ppm. ${ }^{13} \mathrm{C}$ NMR (DMSO-d $\left.6, \delta\right), 166.4(\mathrm{C}=\mathrm{O}), 165.4(\mathrm{C}=\mathrm{O}), 137.3$, 
133.1, 132.5, 131.9, 130.0, 129.2, 129.1, 128.0 ppm. MS (ES $\left.{ }^{+}, \mathrm{m} / \mathrm{z}\right), 297[\mathrm{M}+\mathrm{Na}]^{+} . \mathrm{MS}(\mathrm{ES}-\mathrm{m} / \mathrm{z}), 273$ $[\mathrm{M}-\mathrm{H}]^{+}$.

N'- Benzoyl-4-bromobenzohydrazide (1g). A white solid (69\%, 2.18 g); m.p. 207-209 C. Selected IR $\left(\mathrm{KBr}, \mathrm{cm}^{-1}\right)$ : 3192(s), 3009(w), 1600(vs), 1576(s), 1561(s), 1461(s), 1264(s), 706(m). ${ }^{1} \mathrm{H}$ NMR (DMSO$\left.\mathrm{d}_{6}, \delta\right), 10.58(\mathrm{~s}, 2 \mathrm{H}, \mathrm{NH}), 7.91(\mathrm{~m}, 3 \mathrm{H}, \mathrm{ArH}), 7.77(\mathrm{~m}, 2 \mathrm{H}, \mathrm{ArH}), 7.55(\mathrm{~m}, 4 \mathrm{H}, \mathrm{ArH}) \mathrm{ppm} .{ }^{13} \mathrm{C} \mathrm{NMR}$ $\left(\mathrm{DMSO}_{-} \mathrm{d}_{6}, \delta\right), 166.4(\mathrm{C}=\mathrm{O}), 165.6(\mathrm{C}=\mathrm{O}), 133.0,132.5,132.2,130.1,129.9,129.1,128.0,126.3$ ppm. MS (CI, m/z), $319[\mathrm{M}+\mathrm{H}]^{+}$.

$N^{\prime}$-Benzoyl-4-fluorobenzohydrazide (1h). A white solid $\left(81 \%, 2.06\right.$ g); m.p. $228-230^{\circ} \mathrm{C}$. Selected IR $\left(\mathrm{KBr}, \mathrm{cm}^{-1}\right)$ : 3209(s), 3011(s), 1674(s), 1635(vs), 1605(s), 1538(s), 1504(s), 1283(s), 1240(s), 1160(m), 849(m), 688(m), 594(m), 541(m). ${ }^{1} \mathrm{H}$ NMR (DMSO-d $\left.6, \delta\right), 10.57$ (s, 2H, NH), 8.02 (m, 2H, ArH), 7.95 (m, 2H, ArH), 7.56 (m, 3H, ArH), 7.38 (m, 2H, ArH) ppm. ${ }^{13} \mathrm{C}$ NMR (DMSO-d 6 , $), 166.4(\mathrm{C}=\mathrm{O}), 165.4$ $(\mathrm{C}=\mathrm{O}), 163.0,133.1,132.5,139.7,129.1,128.0,116.3,116.0 \mathrm{ppm} . \mathrm{MS}\left(\mathrm{ES}^{+}, \mathrm{m} / \mathrm{z}\right), 281[\mathrm{M}+\mathrm{Na}]^{+} . \mathrm{MS}$ $\left(\mathrm{ES}^{-}, \mathrm{m} / \mathrm{z}\right), 257[\mathrm{M}-\mathrm{H}]^{+}$.

N'- Benzoylthiophen-2-carbohydrazide (1i). A white solid (70\%, 1.72 g); m.p. 102-104 ${ }^{\circ}$ C. Selected IR $\left(\mathrm{KBr}, \mathrm{cm}^{-1}\right): 3210(\mathrm{~m}), 3020(\mathrm{~m}), 1715(\mathrm{~m}), 1652(\mathrm{~s}), 1536(\mathrm{~s}), 1270(\mathrm{~s}), 709(\mathrm{~m}) .{ }^{1} \mathrm{H}$ NMR (DMSO-d 6 , $\delta$ ), $9.80(\mathrm{~s}, 2 \mathrm{H}, \mathrm{NH}), 9.81(\mathrm{~s}, 2 \mathrm{H}, \mathrm{NH}), 9.00(\mathrm{~m}, 3 \mathrm{H}), 7.72(\mathrm{~m}, 3 \mathrm{H}), 7.13(\mathrm{~m}, 2 \mathrm{H}) .{ }^{13} \mathrm{C}$ NMR (DMSO-d,$\left.\delta\right)$, $161.7(\mathrm{C}=\mathrm{O}), 139.0(\mathrm{C}=\mathrm{O}), 130.9,128.5,128.0, \mathrm{MS}(\mathrm{CI}, \mathrm{m} / \mathrm{z}), 247[\mathrm{M}+\mathrm{H}]^{+}$.

Ethyl 2-benzoylhydrazinecarboxylate (1j). A white paste $(90 \%, 1.85 \mathrm{~g})$; Selected IR $\left(\mathrm{KBr}, \mathrm{cm}^{-1}\right)$ : 3208(m), 3021(m), 1711(s), 1650(s), 1530(m), 1326(m), 1267(s), 707(s). ${ }^{1} \mathrm{H}$ NMR (DMSO-d $\left.6, \delta\right), 8.81$ $(\mathrm{s}, 2 \mathrm{H}, \mathrm{NH}), 8.10(\mathrm{~m}, 2 \mathrm{H}, \operatorname{ArH}), 7.80(\mathrm{~m}, 1 \mathrm{H}, \operatorname{ArH}), 7.48(\mathrm{~m}, 2 \mathrm{H}, \operatorname{ArH}), 4.14(\mathrm{q}, J(\mathrm{H}, \mathrm{H})=7.2 \mathrm{~Hz}, 2 \mathrm{H}$, $\left.\mathrm{OCH}_{2}\right), 1.23\left(\mathrm{t}, J(\mathrm{H}, \mathrm{H})=7.2 \mathrm{~Hz}, 3 \mathrm{H}, \mathrm{CH}_{3}\right) \mathrm{ppm} .{ }^{13} \mathrm{C}$ NMR $\left(\mathrm{DMSO}-\mathrm{d}_{6}, \delta\right), 171.1(\mathrm{C}=\mathrm{O}), 167.3(\mathrm{C}=\mathrm{O})$, 133.7, 130.1, 127.4, 62.5, 14.2 ppm. MS (CI, m/z), $209[\mathrm{M}+\mathrm{H}]^{+}$.

$N^{\prime}$-Benzoylfuran-2-carbohydrazide (1k). A white solid $(81 \%, 1.85$ g); m.p. 208-210 C. Selected IR $\left(\mathrm{KBr}, \mathrm{cm}^{-1}\right):$ 3198(s), 1672(m), 1634(vs), 1592(m), 1580(m), 1525(m), 1471(m), 1291(s), 1221(m), 1159(m), 1010(m), 846(m), 754(m), 703(m), 689(m), 594(m), 540(m). ${ }^{1} \mathrm{H}$ NMR (DMSO-d $\left.6, \delta\right), 10.46(\mathrm{~s}$, 2H, NH), 7.95 (m, 3H, ArH), 7.55 (m, 3H, ArH\&FurH), 7.28 (d, 1H, FurH), 6.69 (t, 1H, FurH) ppm. ${ }^{13} \mathrm{C}$ NMR (DMSO-d 6 , $\delta), 166.4(\mathrm{C}=\mathrm{O}), 157.9(\mathrm{C}=\mathrm{O}), 146.9,146.4,133.0,132.5,129.1,128.0,115.2,112.5$ ppm. MS $\left(\mathrm{ES}^{+}, \mathrm{m} / \mathrm{z}\right), 253[\mathrm{M}+\mathrm{Na}]^{+} . \mathrm{MS}\left(\mathrm{ES}^{-}, \mathrm{m} / \mathrm{z}\right), 229[\mathrm{M}-\mathrm{H}]^{+}$. 
$N^{\prime}$-(4-Methylbenzoyl)furan)-2-carbohydrazide (1l). A white solid (65\%, $\left.1.57 \mathrm{~g}\right)$; m.p. 225-227 ${ }^{\circ}$. Selected IR (KBr, $\left.\mathrm{cm}^{-1}\right)$ : 3173(m), 3011(m), 1673(m), 1631(vs), 1592(m), 1527(m), 1504(m), 1471(m), 1286, 1217(m), 1186(m), 1120(m), 1020(m), 851(m), 746(s), 595(s), 540(m). ${ }^{1} \mathrm{H}$ NMR (DMSO-d $\left.6, \delta\right)$, $10.37(\mathrm{~s}, 2 \mathrm{H}, \mathrm{NH}), 7.91(\mathrm{~d}, 1 \mathrm{H}, \mathrm{FurH}), 7.81(\mathrm{~d}, J(\mathrm{H}, \mathrm{H})=6.6 \mathrm{~Hz}, 2 \mathrm{H}, \mathrm{ArH}), 7.31(\mathrm{~d}, J(\mathrm{H}, \mathrm{H})=6.6 \mathrm{~Hz}, 2 \mathrm{H}$, ArH), 7.26 (d, 1H, FurH), 6.68 (m, 1H, FurH), 2.37 (s, 3H, $\mathrm{CH}_{3}$ ) ppm. ${ }^{13} \mathrm{C}$ NMR (DMSO-d 6 , $\left.\delta\right), 166.3$ $(\mathrm{C}=\mathrm{O}), 158.0(\mathrm{C}=\mathrm{O}), 146.9,146.3,142.5,130.2,129.6,128.1,115.1,112.4,21.6 \mathrm{ppm} . \mathrm{MS}\left(\mathrm{ES}^{+}, \mathrm{m} / \mathrm{z}\right)$, $267[\mathrm{M}+\mathrm{Na}]^{+}$.

$N^{\prime}$-(4-Bromobenzoyl)furan-2-carbohydrazide (1m). A white solid (63\%, $\left.1.95 \mathrm{~g}\right)$; m.p. 191-193 ${ }^{\circ} \mathrm{C}$. Selected IR (KBr, cm $\left.{ }^{-1}\right):$ 3167(w), 3004(w), 1637(vs), 1592(s), 1520(m), 1483(m), 1289(m), 1010(m), 840(m), 747(m), 594(m). ${ }^{1} \mathrm{H}$ NMR (DMSO-d $\left.6, \delta\right), 10.57$ (s, 2H, NH), $7.94(\mathrm{~d}, J(\mathrm{H}, \mathrm{H})=10.2 \mathrm{~Hz}, 1 \mathrm{H}$, FurH), $7.85(\mathrm{~d}, J(\mathrm{H}, \mathrm{H})=8.2 \mathrm{~Hz}, 2 \mathrm{H}, \operatorname{ArH}), 7.74(\mathrm{~d}, J(\mathrm{H}, \mathrm{H})=8.2 \mathrm{~Hz}, 2 \mathrm{H}, \operatorname{ArH}), 7.27(\mathrm{~d}, J(\mathrm{H}, \mathrm{H})=10.2$ $\mathrm{Hz}, 1 \mathrm{H}$, FurH), 6.69 (m, 1H, FurH) ppm. ${ }^{13} \mathrm{C}$ NMR (DMSO-d 6 , $), 165.5(\mathrm{C}=\mathrm{O}), 157.9(\mathrm{C}=\mathrm{O}), 148.4$, $146.4,132.5,132.2,130.1,126.3,115.3,112.5$ ppm. MS $\left(\mathrm{ES}^{+}, \mathrm{m} / \mathrm{z}\right), 333[\mathrm{M}+\mathrm{Na}]^{+} . \mathrm{MS}(\mathrm{ES}-\mathrm{m} / \mathrm{z}), 309$ $[\mathrm{M}-\mathrm{H}]^{+}$.

4-Methoxy- $N^{\prime}$-(4-methylbenzoyl)benzohydrazide (1n). A white solid (69\%, 1.94 g); m.p. 214-216 ${ }^{\circ}$. Selected IR (KBr, cm $\left.{ }^{-1}\right): 3214(\mathrm{~s}), 3010(\mathrm{w}), 2844(\mathrm{w}), 1599(\mathrm{vs}), 1561(\mathrm{~m}), 1512(\mathrm{~m}), 1469(\mathrm{~m}), 1440(\mathrm{~m})$, 1255(s), 1177(m), 1028(m), 840(m), 743(m), 598(m). ( ${ }^{1} \mathrm{H}_{\mathrm{NMR}}\left(\mathrm{DMSO}_{6}, \delta\right), 10.35$ (s, 2H, NH), 7.92 $(\mathrm{d}, J(\mathrm{H}, \mathrm{H})=8.5 \mathrm{~Hz}, 2 \mathrm{H}, \operatorname{ArH}), 7.83(\mathrm{~d}, J(\mathrm{H}, \mathrm{H})=8.5 \mathrm{~Hz}, 2 \mathrm{H}, \operatorname{ArH}), 7.31(\mathrm{~d}, J(\mathrm{H}, \mathrm{H})=8.5 \mathrm{~Hz}, 2 \mathrm{H}, \operatorname{ArH})$, $7.05(\mathrm{~d}, J(\mathrm{H}, \mathrm{H})=8.5 \mathrm{~Hz}, 2 \mathrm{H}, \mathrm{ArH}), 3.85\left(\mathrm{~s}, 3 \mathrm{H}, \mathrm{OCH}_{3}\right), 2.37\left(\mathrm{~s}, 3 \mathrm{H}, \mathrm{CH}_{3}\right) \mathrm{ppm} .{ }^{13} \mathrm{C} \mathrm{NMR}\left(\mathrm{DMSO}-\mathrm{d}_{6}\right.$,

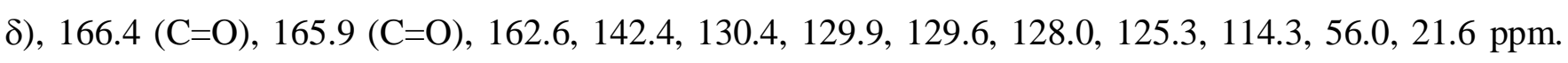
$\mathrm{MS}\left(\mathrm{ES}^{+}, \mathrm{m} / \mathrm{z}\right), 307[\mathrm{M}+\mathrm{Na}]^{+}$.

$N^{\prime}$-Benzoyl-4-methoxybenzohydrazide (1p). A white solid (77\%, 2.07 g); m.p. 188-190 C. Selected IR $\left(\mathrm{KBr}, \mathrm{cm}^{-1}\right)$ : 3206(s), 3005(s), 1632(s), 1607(s), 1542(m), 1505(s), 1260(s), 1175(m), 1031(m), 842(m), 691(m). ${ }^{1} \mathrm{H}$ NMR (DMSO-d 6 , $), 10.42$ (s, 2H, NH), 7.92 (m, 4H, ArH), 7.55 (m, 3H, ArH), 7.07 (m, 2H, ArH), 3.41 (s, 3H, $\left.\mathrm{OCH}_{3}\right)$ ppm. ${ }^{13} \mathrm{C}$ NMR (DMSO-d $\left.6, \delta\right), 166.5(\mathrm{C}=\mathrm{O}), 165.9(\mathrm{C}=\mathrm{O}), 133.3,132.4$, 130.0, 129.1, 128.0, 125.3, 114.3, 56.0 $\left(\mathrm{OCH}_{3}\right) \mathrm{ppm} . \mathrm{MS}(\mathrm{CI}, \mathrm{m} / \mathrm{z}), 271[\mathrm{M}+\mathrm{H}]^{+}$.

4-Chloro- $N$ '-(4-methylbenzoyl)benzohydrazide (1r). A white solid (68\%, 1.95 g); m.p. 255-256 C. Selected IR (KBr, $\left.\mathrm{cm}^{-1}\right)$ : 3193(s), 3014(w), 1599(vs), 1563(s), 1511(m), 1463(s), 1267(m), 1225(m), 1090(m), 1012(m), 850(m), 741(m), 663(m), 603(m), 462(m). ${ }^{1} \mathrm{H}$ NMR (DMSO-d $\left.6, \delta\right), 10.53(\mathrm{~s}, 2 \mathrm{H}$, $\mathrm{NH}), 7.94(\mathrm{~d}, J(\mathrm{H}, \mathrm{H})=7.7 \mathrm{~Hz}, 2 \mathrm{H}, \mathrm{ArH}), 7.74(\mathrm{~d}, J(\mathrm{H}, \mathrm{H})=7.2 \mathrm{~Hz}, 2 \mathrm{H}, \operatorname{ArH}), 7.61(\mathrm{~d}, J(\mathrm{H}, \mathrm{H})=7.2 \mathrm{~Hz}$, 
$2 \mathrm{H}, \operatorname{ArH}), 7.33(\mathrm{~d}, J(\mathrm{H}, \mathrm{H})=7.7 \mathrm{~Hz}, 2 \mathrm{H}, \mathrm{ArH}), 2.38\left(\mathrm{~s}, 3 \mathrm{H}, \mathrm{CH}_{3}\right)$ ppm. ${ }^{13} \mathrm{C} \mathrm{NMR}\left(\mathrm{DMSO}-\mathrm{d}_{6}, \delta\right), 166.3$ $(\mathrm{C}=\mathrm{O}), 165.4(\mathrm{C}=\mathrm{O}), 142.5,137.3,131.9,130.2,130.0,129.6,129.3,128.1,21.6 \mathrm{ppm} . \mathrm{MS}\left(\mathrm{ES}^{+}, \mathrm{m} / \mathrm{z}\right)$, $311[\mathrm{M}+\mathrm{Na}]^{+} . \mathrm{MS}\left(\mathrm{ES}^{-}, \mathrm{m} / \mathrm{z}\right), 287[\mathrm{M}-\mathrm{H}]^{+}$.

4-Fluoro- $N^{\prime}$-(4-methylbenzoyl)benzohydrazide (1s). A white solid (57\%, 1.55 g); m.p. 224-225 C. Selected IR (KBr, $\left.\mathrm{cm}^{-1}\right)$ : 3202(m), 3013(w), 1605(vs), 1584(s), 1511(m), 1461(s), 1267(m), 1225(m), 1155(m), 851(m), 742(m), 661(m), 600(m). ${ }^{1} \mathrm{H}$ NMR (DMSO-d 6 , $), 10.49$ (s, 2H, NH), $8.01(\mathrm{~d}, J(\mathrm{H}, \mathrm{H})$ $=6.3 \mathrm{~Hz}, 2 \mathrm{H}, \mathrm{ArH}), 7.83(\mathrm{~d}, J(\mathrm{H}, \mathrm{H})=6.3 \mathrm{~Hz}, 2 \mathrm{H}, \mathrm{ArH}), 7.34(\mathrm{~m}, 4 \mathrm{H}, \mathrm{ArH}), 2.37\left(\mathrm{~s}, 3 \mathrm{H}, \mathrm{CH}_{3}\right) \mathrm{ppm} .{ }^{13} \mathrm{C}$ NMR (DMSO-d $\left.{ }_{6}, \delta\right), 166.3(\mathrm{C}=\mathrm{O}), 165.4(\mathrm{C}=\mathrm{O}), 162.9,142.5,130.8,130.7,130.3,129.6,128.1,116.3$, 115.9, 21.6 ppm. MS $\left(\mathrm{ES}^{+}, \mathrm{m} / \mathrm{z}\right), 295[\mathrm{M}+\mathrm{Na}]^{+}, 273[\mathrm{M}+\mathrm{H}]^{+}$.

4-Bromo- $N^{\prime}$-(4-methoxybenzoyl)benzohydrazide (1t). A white solid (81\%, 2.80 g); m.p. 240-242 ${ }^{\circ}$ C. Selected IR (KBr, cm $\left.{ }^{-1}\right)$ : 3209(m), 3009(w), 1600(vs), 1560(m), 1467(s), 1255(s), 1178(m), 1031(m), 847(m), 744(m), 609(m). ${ }^{1} \mathrm{H}$ NMR (DMSO-d $\left.6, \delta\right), 10.54$ (s, 2H, NH), 7.90 (m, 4H, ArH), $7.76(\mathrm{~d}, J(\mathrm{H}, \mathrm{H})$ $=7.7 \mathrm{~Hz}, 2 \mathrm{H}, \mathrm{ArH}), 7.05(\mathrm{~d}, J(\mathrm{H}, \mathrm{H})=7.7 \mathrm{~Hz}, 2 \mathrm{H}, \mathrm{ArH}), 3.83\left(\mathrm{~s}, 3 \mathrm{H}, \mathrm{OCH}_{3}\right) \mathrm{ppm} .{ }^{13} \mathrm{C} \mathrm{NMR}\left(\mathrm{DMSO}-\mathrm{d}_{6}\right.$,

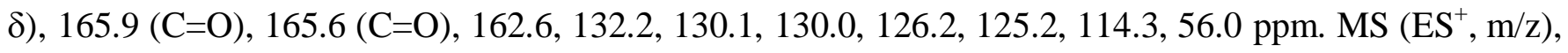
$371[\mathrm{M}+\mathrm{Na}]^{+}$.

General procedure for the synthesis of 2,5-disubstituted 1,3,4-selenadiazoles (2a-t): A mixture of 1,2-diacylhydrazines $(1.0 \mathrm{mmol})$ and Woollins' reagent $(0.54 \mathrm{~g}, 1.0 \mathrm{mmol})$ in $20 \mathrm{ml}$ of dry toluene was refluxed for $7 \mathrm{~h}$. The red suspension disappeared and a brown suspension was formed along with some grey elemental selenium. After cooling to room temperature the mixture was dried in vacuo to remove toluene. The residue was then dissolved in dichloromethane and purified by silica gel (1:5 ethyl acetate / dichloromethane as eluent) to give the corresponding target product.

2,5-Diphenyl-1,3,4-selenadiazole (2a). A yellow solid (98\%, 0.28 g); m.p. 116-118 $\mathrm{C}$. Selected IR (KBr, $\left.\mathrm{cm}^{-1}\right)$ : 3424(s), 1438(m), 1149(s), 933(m), 691(s), 543(s). ${ }^{1} \mathrm{H}$ NMR $\left(\mathrm{CD}_{2} \mathrm{Cl}_{2}, \delta\right), 7.95$ (m, 4H, ArH), 7.50 $(\mathrm{m}, 6 \mathrm{H}, \mathrm{ArH}) \mathrm{ppm} .{ }^{13} \mathrm{C} \mathrm{NMR}\left(\mathrm{CD}_{2} \mathrm{Cl}_{2}, \delta\right), 174.9(\mathrm{C}=\mathrm{N}), 1431.2,129.3,128.7,126.9 \mathrm{ppm} .{ }^{77} \mathrm{Se} \mathrm{NMR}$ $\left(\mathrm{CD}_{2} \mathrm{Cl}_{2}, \delta\right), 687.13$ ppm. MS (CI, m/z), $287[\mathrm{M}+\mathrm{H}]^{+} . \mathrm{C}_{14} \mathrm{H}_{10} \mathrm{~N}_{2} \mathrm{Se}$ (285.11): Calcd. C 58.98, H 3.54, N 9.83; found C 59.30, H 3.85, N 9.79.

2,5-Bis(4-bromophenyl)-1,3,4-selenadiazole (2b). ). A yellow solid (75\%, 0.33 g); m.p. $210^{\circ} \mathrm{C}$ (Dec.). Selected IR (KBr, cm ${ }^{-1}$ ): 3424(w), 2921(w), 1602(s), 1544(m), 1477(vs), 1397(m), 1266(w), 1090(s), 1072(s), 1009(s), 838(s), 737(s), 520(m). ${ }^{1} \mathrm{H}$ NMR $\left(\mathrm{CD}_{2} \mathrm{Cl}_{2}, \delta\right), 8.06(\mathrm{~d}, J(\mathrm{H}, \mathrm{H})=8.5 \mathrm{~Hz}, 2 \mathrm{H}, \mathrm{ArH}), 7.98$ 
$(\mathrm{d}, J(\mathrm{H}, \mathrm{H})=8.5 \mathrm{~Hz}, 2 \mathrm{H}, \mathrm{ArH}), 7.67(\mathrm{~d}, J(\mathrm{H}, \mathrm{H})=8.5 \mathrm{~Hz}, 2 \mathrm{H}, \operatorname{ArH}), 7.51(\mathrm{~d}, J(\mathrm{H}, \mathrm{H})=8.5 \mathrm{~Hz}, 2 \mathrm{H}, \operatorname{ArH})$ ppm. ${ }^{13} \mathrm{C}$ NMR $\left(\mathrm{CD}_{2} \mathrm{Cl}_{2}, \delta\right), 164.1(\mathrm{C}=\mathrm{N}), 151.7(\mathrm{C}=\mathrm{N}), 138.3,132.6,130.1,129.9,129.6,128.4,128.3$, 126.7, 122.7 ppm. ${ }^{77} \mathrm{Se} \mathrm{NMR}\left(\mathrm{CD}_{2} \mathrm{Cl}_{2}, \delta\right), 690.60 \mathrm{ppm}$. MS (CI, m/z), $443[\mathrm{M}+\mathrm{H}]^{+} . \mathrm{C}_{14} \mathrm{H}_{8} \mathrm{Br}_{2} \mathrm{~N}_{2} \mathrm{Se}$ (442.91): Calcd. C 37.96, H 1.82, N 6.32; found C 37.66, H 1.91; N 6.08.

2-Phenyl-5-(pyridine-3-yl)-1,3,4-selenadiazole (2c). A greenish yellow solid (51\%, 0.15 g); m.p. 90$92^{\circ} \mathrm{C}$. Selected IR $\left(\mathrm{KBr}, \mathrm{cm}^{-1}\right):$ 3424(s), 1454(m), 1435(m), 1127(m), 1055(m), 761(m), 690(s), 532(s). ${ }^{1} \mathrm{H}$ NMR $\left(\mathrm{CD}_{2} \mathrm{Cl}_{2}, \delta\right), 8.38$ (s, 1H, PyH), 8.13 (d, 1H, PyH), 7.93 (m, 3H, PyH \& ArH), 7.57 (m, 4H, ArH) ppm. ${ }^{13} \mathrm{C}$ NMR $\left(\mathrm{CD}_{2} \mathrm{Cl}_{2}, \delta\right), 149.5(\mathrm{C}=\mathrm{N}), 147.6(\mathrm{C}=\mathrm{N}), 137.3,131.6,129.4,128.9,127.0,124.9$ ppm. ${ }^{77} \mathrm{Se}$ NMR $\left(\mathrm{CD}_{2} \mathrm{Cl}_{2}, \delta\right), 698.88$ ppm. MS (CI, m/z), $287[\mathrm{M}+\mathrm{H}]^{+} . \mathrm{C}_{13} \mathrm{H}_{9} \mathrm{~N}_{3} \mathrm{Se}$ (286.10): Calcd. C 54.56, H 3.17, N 14.68; found C 54.10, H 3.61, N 14.57.

2-(4-Methoxyphenyl)-5-(pyridine-3-yl)-1,3,4-selenadiazole (2d). A yellow solid (58\%, 0.18 g); m.p. 98-100 ${ }^{\circ}$ C. Selected IR (KBr, $\left.\mathrm{cm}^{-1}\right): 3425(\mathrm{~m}), 3049(\mathrm{~m}), 1454(\mathrm{~m}), 1434(\mathrm{~s}), 1140(\mathrm{~m}), 1081(\mathrm{~m}), 958(\mathrm{~m})$, 761(m), 690(s), 534(s). ${ }^{1} \mathrm{H}$ NMR $\left(\mathrm{CD}_{2} \mathrm{Cl}_{2}, \delta\right), 9.54$ (s, 1H, PyH), 9.01 (d, 1H, PyH), 8.88 (d, 1H, PyH), $8.01(\mathrm{~m}, 3 \mathrm{H}, \mathrm{PyH} \& \mathrm{ArH}), 7.47$ (m, 4H, PyH \& ArH), 3.86 (s, 3H, OCH 3$)$ ppm. ${ }^{13} \mathrm{C} \mathrm{NMR}\left(\mathrm{CD}_{2} \mathrm{Cl}_{2}, \delta\right)$, $144.0(\mathrm{C}=\mathrm{N}), 142.6(\mathrm{C}=\mathrm{N}), 132.0,129.4,129.0,128.4,128.2,127.9,127.2,55.8 \mathrm{ppm} .{ }^{77} \mathrm{Se}$ NMR $\left(\mathrm{CD}_{2} \mathrm{Cl}_{2}, \delta\right), 714.61$ ppm. MS (CI, m/z), $318[\mathrm{M}+\mathrm{H}]^{+} . \mathrm{C}_{14} \mathrm{H}_{12} \mathrm{~N}_{3} \mathrm{OSe}$ (317.14): Calcd. C 53.18, H 3.51, N 13.29; found C 53.51, H 3.61, N 13.57.

2-Phenyl-5-p-tolyl-1,3,4-selenadiazole (2e). A yellow solid (90\%, 0.27 g); m.p. 102-104 ${ }^{\circ}$ C. Selected IR $\left(\mathrm{KBr}, \mathrm{cm}^{-1}\right):$ 2918(w), 1607(m), 1546(m), 1494(m), 1441(s), 1258(m), 1061(s), 962(m), 817(s), 757(m), 685(s), 578(m). ${ }^{1} \mathrm{H}$ NMR $\left(\mathrm{CD}_{2} \mathrm{Cl}_{2}, \delta\right), 8.12(\mathrm{~d}, J(\mathrm{H}, \mathrm{H})=7.2 \mathrm{~Hz}, 2 \mathrm{H}, \mathrm{ArH}), 7.83(\mathrm{~d}, J(\mathrm{H}, \mathrm{H})=7.9 \mathrm{~Hz}, 2 \mathrm{H}$, $\operatorname{ArH}), 7.49(\mathrm{~m}, 3 \mathrm{H}, \mathrm{ArH}), 7.27(\mathrm{~d}, J(\mathrm{H}, \mathrm{H})=7.9 \mathrm{~Hz}, 2 \mathrm{H}, \mathrm{ArH}), 2.39\left(\mathrm{~s}, 3 \mathrm{H}, \mathrm{CH}_{3}\right) \mathrm{ppm} .{ }^{13} \mathrm{C} \mathrm{NMR}$ $\left(\mathrm{CD}_{2} \mathrm{Cl}_{2}, \delta\right), 164.8(\mathrm{C}=\mathrm{N}), 164.4(\mathrm{C}=\mathrm{N}), 141.9,131.6,131.0,129.9,129.2,128.4,126.8,21.4 \mathrm{ppm} .{ }^{77} \mathrm{Se}$ NMR $\left(\mathrm{CD}_{2} \mathrm{Cl}_{2}, \delta\right), 683.01$ ppm. MS (CI, m/z), $301[\mathrm{M}+\mathrm{H}]^{+} . \mathrm{C}_{15} \mathrm{H}_{12} \mathrm{~N}_{2} \mathrm{Se}$ (300.02): Calcd. C 60.21, H 4.04, N 9.36; found C 60.31, H 4.29, N 9.51.

2-(4-Chlorophenyl)-5-phenyl-1,3,4-selenadiazole (2f). A yellow solid (97\%, $0.31 \mathrm{~g})$; m.p. 198-199 ${ }^{\circ}$. Selected IR (KBr, $\left.\mathrm{cm}^{-1}\right)$ : 3424(w), 1590(m), 1494(m), 1440(vs), 1418(s), 1238(m), 1089(s), 1062(s), 1010(m), 965(m), 845(m), 820(m), 762(s), 686(m), 661(m), 578(m). ${ }^{1} \mathrm{H}$ NMR $\left(\mathrm{CD}_{2} \mathrm{Cl}_{2}, \delta\right), 7.95(\mathrm{~m}, 4 \mathrm{H}$, ArH), 7.47 (m, 5H, ArH) ppm. ${ }^{13} \mathrm{C}$ NMR $\left(\mathrm{CD}_{2} \mathrm{Cl}_{2}, \delta\right), 136.9(\mathrm{C}=\mathrm{N}), 133.1(\mathrm{C}=\mathrm{N}), 131.7,131.3,129.9$, 129.5, 129.3, 128.7 ppm. ${ }^{77} \mathrm{Se} \mathrm{NMR}\left(\mathrm{CD}_{2} \mathrm{Cl}_{2}, \delta\right), 688.61 \mathrm{ppm} . \mathrm{MS}\left(\mathrm{ESI}^{+}, \mathrm{m} / \mathrm{z}\right), 343[\mathrm{M}+\mathrm{Na}]^{+}$. $\mathrm{C}_{14} \mathrm{H}_{9} \mathrm{ClN}_{2} \mathrm{Se}$ (319.56): Calcd. C 52.61, H 2.84, N 8.76; found C 52.56, H 2.49, N 8.49. 
2-(4-Bromophenyl)-5-phenyl-1,3,4-selenadiazole (2g). A greenish yellow solid (95\%, 0.35 g); m.p. 90$92^{\circ} \mathrm{C}$. Selected IR (KBr, cm $\left.{ }^{-1}\right): 3449(\mathrm{w}), 1583(\mathrm{~m}), 1492(\mathrm{~m}), 1439(\mathrm{~s}), 1417(\mathrm{~m}), 1256(\mathrm{~m}), 1063(\mathrm{~s}), 824(\mathrm{~s})$, 760(s), 688(s), 577(m). ${ }^{1} \mathrm{H}$ NMR $\left(\mathrm{CD}_{2} \mathrm{Cl}_{2}, \delta\right), 7.94$ (d, 2H, ArH), 7.83 (d, 2H, ArH), 7.62 (m, 2H, ArH), $7.50(\mathrm{~d}, 3 \mathrm{H}, \mathrm{ArH}) \mathrm{ppm} .{ }^{13} \mathrm{C}$ NMR $\left(\mathrm{CD}_{2} \mathrm{Cl}_{2}, \delta\right), 164.8(\mathrm{C}=\mathrm{N}), 164.0(\mathrm{C}=\mathrm{N}), 132.5,131.9,131.3,130.0$, 129.3, 128.7, 128.3, 126.9 ppm. ${ }^{77} \mathrm{Se} \mathrm{NMR}\left(\mathrm{CD}_{2} \mathrm{Cl}_{2}, \delta\right), 688.75 \mathrm{ppm} . \mathrm{MS}(\mathrm{CI}, \mathrm{m} / \mathrm{z}), 365[\mathrm{M}+\mathrm{H}]^{+}$. $\mathrm{C}_{14} \mathrm{H}_{9} \mathrm{BrN}_{2} \mathrm{Se}$ (364.01): Calcd. C 46.18, H 2.49, N 7.69; found C 46.96, H 2.28, N 7.78.

2-(4-Fluorophenyl)-5-phenyl-1,3,4-selenadiazole (2h). A greenish yellow solid (91\%, $0.28 \mathrm{~g})$; m.p. 102-104 ${ }^{\circ}$ C. Selected IR (KBr, $\left.\mathrm{cm}^{-1}\right)$ : 3427(w), 3063(w), 1606(m), 1550(m), 1495(vs), 1445(m), 1415(m), 1234(s), 1152(m), 1072(m), 844(s), 734(s), 687(s), 616(m), 523(m). ${ }^{1} \mathrm{H}$ NMR $\left(\mathrm{CD}_{2} \mathrm{Cl}_{2}, \delta\right), 8.15(\mathrm{~d}, 2 \mathrm{H}$, ArH), 7.96 (d, 2H, ArH), 7.53 (m, 3H, ArH), 7.25 (m, 2H, ArH) ppm. ${ }^{13} \mathrm{C} \mathrm{NMR}\left(\mathrm{CD}_{2} \mathrm{Cl}_{2}, \delta\right), 166.3$ $(\mathrm{C}=\mathrm{N}), 163.8(\mathrm{C}=\mathrm{N}), 131.8,131.2,130.7,129.2,128.7,126.8,117.2,116.2 \mathrm{ppm} .{ }^{77} \mathrm{Se} \mathrm{NMR}\left(\mathrm{CD}_{2} \mathrm{Cl}_{2}, \delta\right)$, 686.62 ppm. MS $\left(\mathrm{ESI}^{+}, \mathrm{m} / \mathrm{z}\right), 327[\mathrm{M}+\mathrm{Na}]^{+} . \mathrm{C}_{14} \mathrm{H}_{9} \mathrm{FN}_{2} \mathrm{Se}$ (303.10): Calcd. C 55.46, H 2.99, N 9.24; found C 55.56, H 2.93, N 9.17.

2-Phenyl-5-(thiophen-2-yl)-1,3,4-selenadiazole (2i). A yellow oil (92\%, $0.31 \mathrm{~g})$. Selected IR (KBr, $\left.\mathrm{cm}^{-1}\right): 3430(\mathrm{w}), 3060(\mathrm{w}), 1608(\mathrm{~m}), 1499(\mathrm{~s}), 1447(\mathrm{~m}), 1420(\mathrm{~m}), 1240(\mathrm{~s}), 1076(\mathrm{~m}), 739(\mathrm{~s}), 690(\mathrm{~s})$, 618(m), 525(m). ${ }^{1} \mathrm{H}$ NMR $\left(\mathrm{CD}_{2} \mathrm{Cl}_{2}, \delta\right), 7.94$ (m, 1H, thiophen-H), 7.75-7.37 (m, 3H, ArH), 7.03 (m, 2H, $\mathrm{ArH}), 6.24\left(\mathrm{~m}, 1 \mathrm{H}\right.$, thiophen-H), $6.12\left(\mathrm{~m}, 1 \mathrm{H}\right.$, thiophen-H) ppm. ${ }^{13} \mathrm{C} \mathrm{NMR}\left(\mathrm{CD}_{2} \mathrm{Cl}_{2}, \delta\right), 166.6(\mathrm{C}=\mathrm{N})$, $164.0(\mathrm{C}=\mathrm{N}), 131.3,131.2,130.8,129.3,128.7,128.3,127.6,127.5,127.1 \mathrm{ppm} .{ }^{77} \mathrm{Se} \mathrm{NMR}\left(\mathrm{CD}_{2} \mathrm{Cl}_{2}, \delta\right)$, 688.24 ppm. MS (CI, m/z), $341[\mathrm{M}+\mathrm{H}]^{+} . \mathrm{C}_{12} \mathrm{H}_{8} \mathrm{~N}_{2} \mathrm{Se}_{2}$ (337.94): Calcd. C 42.63, H 2.39, N 8.28; found C 42.90, H 2.48, N 8.08.

2-Ethoxy-5-phenyl-1,3,4-selenadiazole (2j). A yellow paste $(99 \%, 0.25 \mathrm{~g})$. Selected IR $\left(\mathrm{KBr}, \mathrm{cm}^{-1}\right)$ : 3056(w), 2926(w), 1757(s), 1719(s), 1690(m), 1438(m), 1369(m), 1307(m), 1281(s), 1232(s), 758(m), 686(s), 543(m). ${ }^{1} \mathrm{H}$ NMR $\left(\mathrm{CD}_{2} \mathrm{Cl}_{2}, \delta\right), 8.14$ (m, 2H, ArH), 7.54 (m, 3H, ArH), 4.17 (t, 2H, OCH $), 1.25$ (q, 3H, $\left.\mathrm{CH}_{3}\right)$ ppm. ${ }^{13} \mathrm{C} \mathrm{NMR}\left(\mathrm{CD}_{2} \mathrm{Cl}_{2}, \delta\right), 135.6(\mathrm{C}=\mathrm{N}), 134.4(\mathrm{C}=\mathrm{N}), 133.0,132.4,132.2,131.3,130.8$, 64.1, 13.8 ppm. ${ }^{77} \mathrm{Se} \mathrm{NMR}\left(\mathrm{CD}_{2} \mathrm{Cl}_{2}, \delta\right), 687.45 \mathrm{ppm}$. MS (CI, m/z), $255[\mathrm{M}+\mathrm{H}]^{+} . \mathrm{C}_{10} \mathrm{H}_{10} \mathrm{~N}_{2} \mathrm{OSe}(253.07)$ : Calcd. C 47.44, H 3.98, N 11.07; found C 47.01, H 3.88, N 10.93.

2-(Furan-2-yl)-5-phenyl-1,3,4-selenadiazole (2k). A yellow solid (82\%, 0.23 g); m.p. 100-102 ${ }^{\circ} \mathrm{C}$. Selected IR (KBr, $\left.\mathrm{cm}^{-1}\right)$ : 3137(m), 1634(m), 1582(m), 1487(s), 1453(s), 1421(m), 1259(m), 1222(m), 1058(m), 1018(s), 879(s), 763(vs), 750(vs), 689(vs), 658(s), 588(s), 557(m). ${ }^{1} \mathrm{H} \mathrm{NMR}_{\left(\mathrm{CD}_{2} \mathrm{Cl}_{2}, \delta\right), 7.94}$ 
(m, 2H, ArH), 7.64 (d, 1H, FurH), 7.47 (m, 3H, ArH), 7.17 (d, 1H, FurH), 6.61 (m, 1H, FurH) ppm. ${ }^{13} \mathrm{C}$ NMR $\left(\mathrm{CD}_{2} \mathrm{Cl}_{2}, \delta\right), 145.9(\mathrm{C}=\mathrm{N}), 145.4(\mathrm{C}=\mathrm{N}), 131.9,131.2,129.3,128.7,126.9,114.0,112.8,112.2$, 110.9 ppm. ${ }^{77} \mathrm{Se}$ NMR $\left(\mathrm{CD}_{2} \mathrm{Cl}_{2}, \delta\right), 692.12 \mathrm{ppm}$. MS (ESI $\left.{ }^{+}, \mathrm{m} / \mathrm{z}\right), 299[\mathrm{M}+\mathrm{Na}]^{+} . \mathrm{C}_{12} \mathrm{H}_{8} \mathrm{~N}_{2} \mathrm{OSe}(275.08)$ : Calcd. C 52.38, H 2.93, N 10.18; found C 52.34, H 2.81, N 10.27.

2-(Furan-2-yl)-5-p-tolyl-1,3,4-selenadiazole (2l). A yellow solid (90\%, 0.24 g); m.p. 98-100 ${ }^{\circ}$. Selected IR (KBr, cm $\left.{ }^{-1}\right)$ : 3449(w), 3104(w), 2896(w), 1607(m), 1492(m), 1443(s), 1261(m), 1061(m), 1017(s), 880(m), 819(s), 754(s), 596(m), 558(m). ${ }^{1} \mathrm{H}$ NMR $\left(\mathrm{CD}_{2} \mathrm{Cl}_{2}, \delta\right), 7.79(\mathrm{~m}, 3 \mathrm{H}), 7.26(\mathrm{~m}, 3 \mathrm{H}), 6.61$ (m, 1H), $2.42\left(\mathrm{~s}, 3 \mathrm{H}, \mathrm{CH}_{3}\right)$ ppm. ${ }^{13} \mathrm{C}$ NMR $\left(\mathrm{CD}_{2} \mathrm{Cl}_{2}, \delta\right), 174.1(\mathrm{C}=\mathrm{N}), 163.0(\mathrm{C}=\mathrm{N}), 145.8,145.2,141.9$, 129.9, 128.5, 112.7, 110.7, 21.3 ppm. ${ }^{77} \mathrm{Se} \mathrm{NMR}\left(\mathrm{CD}_{2} \mathrm{Cl}_{2}, \delta\right), 688.42$ ppm. MS (ESI, $\left.\mathrm{m} / \mathrm{z}\right), 313$ $[\mathrm{M}+\mathrm{Na}]^{+} . \mathrm{C}_{13} \mathrm{H}_{10} \mathrm{~N}_{2} \mathrm{OSe}$ (289.10): Calcd. C 57.15, H 3.69, N 10.25; found C 56.75, H 3.54, N 10.14.

2-(4-Bromophenyl)-5-(furan-2-yl)-1,3,4-selenadiazole (2m). A yellow solid (96\%, 0.34 g); m.p. 138$140^{\circ} \mathrm{C}$. Selected IR (KBr, $\left.\mathrm{cm}^{-1}\right): 3449(\mathrm{~m}), 2920(\mathrm{w}), 2848(\mathrm{w}), 1586(\mathrm{~m}), 1489(\mathrm{~s}), 1445(\mathrm{~s}), 1391(\mathrm{~m})$, 1254(m), 1217(m), 1055(m), 1018(m), 879(m), 815(s), 743(s), 591(m), 554(m). ${ }^{1} \mathrm{H}$ NMR $\left(\mathrm{CD}_{2} \mathrm{Cl}_{2}, \delta\right)$, 7.80 (d, 2H, ArH), 7.61 (d, 2H, ArH), 7.78 (d, 1H, FurH), 7.19 (d, 1H, FurH), 6.61 (m, 1H, FurH) ppm. ${ }^{13} \mathrm{C}$ NMR $\left(\mathrm{CD}_{2} \mathrm{Cl}_{2}, \delta\right), 172.7(\mathrm{C}=\mathrm{N}), 163.8(\mathrm{C}=\mathrm{N}), 146.1,145.5,132.5,130.0,128.4,125.4,112.9,111.1$ ppm. ${ }^{77} \mathrm{Se}$ NMR $\left(\mathrm{CD}_{2} \mathrm{Cl}_{2}, \delta\right), 693.47$ ppm. MS (CI, m/z), $355[\mathrm{M}+\mathrm{H}]^{+} . \mathrm{C}_{12} \mathrm{H}_{7} \mathrm{BrN}_{2} \mathrm{OSe}$ (353.97): Calcd. C 40.71, H 1.99, N 7.91; found C 40.96, H 1.92, N 8.01.

2-(4-Methoxyphenyl)-5-p-tolyl-1,3,4-selenadiazole (2n). A yellow solid (90\%, 0.29 g); m.p. 178-180 ${ }^{\circ}$. Selected IR (KBr, cm $\left.{ }^{-1}\right)$ : 3450(w), 2961(w), 1603(s), 1513(m), 1452(s), 1407(m), 1307(m), 1256(vs), 1177(m), 1065(m), 1034(m), 836(s), 817(s), 606(m), 578(m). ${ }^{1} \mathrm{H}$ NMR $\left(\mathrm{CD}_{2} \mathrm{Cl}_{2}, \delta\right), 7.85$ (m, 4H, $\left.\mathrm{ArH}\right)$, $7.27(\mathrm{~d}, J(\mathrm{H}, \mathrm{H})=7.7 \mathrm{~Hz}, 2 \mathrm{H}, \mathrm{ArH}), 6.96(\mathrm{~d}, J(\mathrm{H}, \mathrm{H})=7.7 \mathrm{~Hz}, 2 \mathrm{H}, \mathrm{ArH}), 3.85\left(\mathrm{~s}, 3 \mathrm{H}, \mathrm{OCH}_{3}\right), 2.39(\mathrm{~s}, 3 \mathrm{H}$, $\left.\mathrm{CH}_{3}\right)$ ppm. ${ }^{13} \mathrm{C} \mathrm{NMR}\left(\mathrm{CD}_{2} \mathrm{Cl}_{2}, \delta\right), 174.0(\mathrm{C}=\mathrm{N}), 162.0(\mathrm{C}=\mathrm{N}), 141.7,130.5,130.2,129.9,128.5,126.0$, 114.5, 55.6, 21.3 ppm. ${ }^{77} \mathrm{Se} \mathrm{NMR}\left(\mathrm{CD}_{2} \mathrm{Cl}_{2}, \delta\right), 673.66$ ppm. MS (ESI, $\left.\mathrm{m} / \mathrm{z}\right), 353[\mathrm{M}+\mathrm{Na}]^{+}$. $\mathrm{C}_{16} \mathrm{H}_{14} \mathrm{~N}_{2} \mathrm{OSe}$ (329.17): Calcd. C 58.37, H 4.29, N 8.51; found C 58.51, H 3.86, N, 8.19.

2-(4-Methoxyphenyl)-5-phenyl-1,3,4-selenadiazole (2p). A yellow solid (86\%, 0.27 g); m.p. 130$132^{\circ} \mathrm{C}$. Selected IR $\left(\mathrm{KBr}, \mathrm{cm}^{-1}\right):$ 2936(w), 2831(w), 1604(s), 1513(m), 1438(s), 1253(vs), 1178(m), 1034(m), 763(m). ${ }^{1} \mathrm{H}$ NMR ( $\left.\mathrm{CD}_{2} \mathrm{Cl}_{2}, \delta\right), 7.91$ (m, 4H, ArH), 7.48 (m, 3H, ArH), 6.99 (m, 2H, ArH), 3.85 $\left(\mathrm{s}, 3 \mathrm{H}, \mathrm{OCH}_{3}\right) \mathrm{ppm} .{ }^{13} \mathrm{C} \mathrm{NMR}\left(\mathrm{CD}_{2} \mathrm{Cl}_{2}, \delta\right), 174.5(\mathrm{C}=\mathrm{N}), 173.9(\mathrm{C}=\mathrm{N}), 133.3,131.0,130.2,129.2,128.6$, 126.3, 125.9, 114.6, 55.6 ppm. ${ }^{77} \mathrm{Se} \mathrm{NMR}\left(\mathrm{CD}_{2} \mathrm{Cl}_{2}, \delta\right), 677.36 \mathrm{ppm} . \mathrm{MS}(\mathrm{CI}, \mathrm{m} / \mathrm{z}), 317[\mathrm{M}+\mathrm{H}]^{+}$. $\mathrm{C}_{15} \mathrm{H}_{12} \mathrm{~N}_{2} \mathrm{OSe}$ (315.14): Calcd. C 57.15, H 3.04, N 8.89; found C 56.54, H 3.62, N 8.94. 
2-(4-Chlorophenyl)-5-p-tolyl-1,3,4-selenadiazole (2r). A yellow solid (83\%, $0.27 \mathrm{~g})$; m.p. 184-186 ${ }^{\circ}$. Selected IR (KBr, cm $\left.{ }^{-1}\right)$ : 3453(w), 3074(w), 2914(w), 1601(m), 1588(m), 1441(s), 1431(s), 1396(m), 1261(m), 1073(m), 817(vs), 577(m), 470(m). ${ }^{1} \mathrm{H}$ NMR $\left(\mathrm{CD}_{2} \mathrm{Cl}_{2}, \delta\right), 8.05(\mathrm{~m}, 4 \mathrm{H}, \mathrm{ArH}), 7.51(\mathrm{~d}, J(\mathrm{H}, \mathrm{H})$ $=6.9 \mathrm{~Hz}, 2 \mathrm{H}, \mathrm{ArH}), 7.34(\mathrm{~d}, J(\mathrm{H}, \mathrm{H})=6.9 \mathrm{~Hz}, 2 \mathrm{H}, \mathrm{ArH}), 2.48\left(\mathrm{~s}, 3 \mathrm{H}, \mathrm{CH}_{3}\right) \mathrm{ppm} .{ }^{13} \mathrm{C} \mathrm{NMR}\left(\mathrm{CD}_{2} \mathrm{Cl}_{2}, \delta\right)$, $164.9(\mathrm{C}=\mathrm{N}), 163.6(\mathrm{C}=\mathrm{N}), 142.7,137.7,129.9,129.5,128.6,128.1,126.8,122.8,21.4$ ppm. ${ }^{77} \mathrm{Se}$ NMR $\left(\mathrm{CD}_{2} \mathrm{Cl}_{2}, \delta\right), 684.21$ ppm. MS (ESI, m/z), 357 [M+Na $]^{+} . \mathrm{C}_{15} \mathrm{H}_{11} \mathrm{ClN}_{2} \mathrm{Se}$ (333.59): Calcd. C 53.99, H 3.32, N 8.40; found C 54.06, H 3.28, N 8.78.

2-(4-Fluorophenyl)-5-p-tolyl-1,3,4-selenadiazole (2s). A greenish yellow solid (79\%, $0.25 \mathrm{~g})$; m.p. 149-151 ${ }^{\circ} \mathrm{C}$. Selected IR (KBr, cm $\left.{ }^{-1}\right)$ : 3062(w), 2920(w), 1607(m), 1494(vs), 1228(s), 1158(m), 1069(m), 1012(m), 963(m), 846(m), 818(m), 741(m), 638(m), 500(m). ${ }^{1} \mathrm{H}$ NMR $\left(\mathrm{CD}_{2} \mathrm{Cl}_{2}, \delta\right), 8.10$ (m, 4H, ArH), $7.27(\mathrm{~m}, 4 \mathrm{H}, \mathrm{ArH}), 2.61$ (s, 3H, $\left.\mathrm{CH}_{3}\right) \mathrm{ppm} .{ }^{13} \mathrm{C} \mathrm{NMR}\left(\mathrm{CD}_{2} \mathrm{Cl}_{2}, \delta\right), 166.6(\mathrm{C}=\mathrm{N}), 163.0(\mathrm{C}=\mathrm{N}), 142.6$, $130.7,129.9,129.2,128.6,126.8,116.6,116.2,21.4$ ppm. ${ }^{77} \mathrm{Se} \mathrm{NMR}\left(\mathrm{CD}_{2} \mathrm{Cl}_{2}, \delta\right), 682.36 \mathrm{ppm} . \mathrm{MS}$ $\left(\mathrm{ESI}^{+}, \mathrm{m} / \mathrm{z}\right), 341[\mathrm{M}+\mathrm{Na}]^{+} . \mathrm{C}_{15} \mathrm{H}_{11} \mathrm{FN}_{2} \mathrm{Se}$ (317.13): Calcd. C 56.79, H 3.50, N 8.83; found C 56.66, H 3.28, N 8.98.

2-(4-Bromophenyl)-5-(4-methoxyphenyl)-1,3,4-selenadiazole (2t). A yellow solid (75\%, 0.30 g); m.p. 148-150 ${ }^{\circ}$ C. Selected IR (KBr, cm $\left.{ }^{-1}\right)$ : 2966(w), 2838(w), 1615(s), 1494(s), 1478(m), 1307(m), 1258(s), 1126(m), 1076(m), 1029(m), 1009(m), 839(s), 742(s), 503(m). ${ }^{1} \mathrm{H}$ NMR $\left(\mathrm{CD}_{2} \mathrm{Cl}_{2}, \delta\right), 8.03$ (m, 4H, ArH), $7.68(\mathrm{~d}, J(\mathrm{H}, \mathrm{H})=8.8 \mathrm{~Hz}, 2 \mathrm{H}, \mathrm{ArH}), 7.04(\mathrm{~d}, J(\mathrm{H}, \mathrm{H})=8.8 \mathrm{~Hz}, 2 \mathrm{H}, \mathrm{ArH}), 3.87\left(\mathrm{~s}, 3 \mathrm{H}, \mathrm{OCH}_{3}\right) \mathrm{ppm} .{ }^{13} \mathrm{C}$ NMR $\left(\mathrm{CD}_{2} \mathrm{Cl}_{2}, \delta\right), 166.2(\mathrm{C}=\mathrm{N}), 163.5(\mathrm{C}=\mathrm{N}), 132.4,130.3,128.6,128.2,126.0,123.2,116.3,114.6$, 55.6 ppm. ${ }^{77} \mathrm{Se} \mathrm{NMR}\left(\mathrm{CD}_{2} \mathrm{Cl}_{2}, \delta\right), 678.61 \mathrm{ppm}$. MS (CI, m/z), $395[\mathrm{M}+\mathrm{H}]^{+} . \mathrm{C}_{15} \mathrm{H}_{11} \mathrm{BrN}_{2} \mathrm{OSe}(394.04)$ : Calcd. C 45.71, H 2.81, N 7.11; found C 45.96, H 2.68, N 7.31.

\section{Acknowledgements}

The authors are grateful to the University of St Andrews and the Engineering and Physical Science Research Council (EPSRC, U.K.) for financial support.

[1] C. J. Lowig, Pogg. Ann. 1836, 37, 552.

[2] a) T. Uemoto, Adv. Heterocycl. Chem. 1995, 64, 323-339; b) V. P. Litvnov, V. D. Diachenko, Russ. Chem. Rev. 1997, 66, 923-951; c) C. Paulmier, Selenium Reagents and Intermediates in Organic 
Synthesis, Pergamom Press, Oxford, 1986; d) T. Back, Organoselenium Chemistry. A Practical Approach, Oxford Press, Oxford 1999; e) J. Mlochowski, Phosphorus, Sulfur, Silicon 1998, 136138, 191; f) T. Wirth, Angew. Chem. Int. Ed. 2000, 39, 3742-3751; g) J. Mlochowski, M. Brzaszez, M. Giurg, J. Palus, H. Wojtowicz, Eur. J. Org. Chem. 2003, 4329-4339; h) G. Mugesh, W.-W. Du Mont, H. Sies, Chem. Rev. 2001, 101, 2125-2179; i) S. Garcia, Curr. Med. Chem. 2004, 11, 16571665 .

[3] a) H. N. Dogan, A. Duran, S. Rollas, G. Sener, M. K. Uysalb and D. Gulenc, Bioorg. Med. Chem. 2002, 10, 2893-2896; b) S. Schenone, O. Bruno, A. Ranise, F. Bondavalli, W. Filippelli, G. Falcone, L. Giordano and M. R. Vitelli, Bioorg. Med. Chem. 2001, 9, 2149-2153; c) J. Y. Chou, X. Y. Lai, S. L. Pan, G. M. Jow, J. W. Chen and J. H. Guh, Biochem. Pharm. 2003, 66, 115-117.

[4] a) M. Saro, T. Kamita, K. Nakadera and K. I. Mukaida, Eur. Polym. J. 1995, 31, 395-400; b) F. Bentiss, M. Lagrenee, J. P. Wignacourt and E. M. Holt, Polyhedron 2002, 21, 403-408; c) J. D. E. T. Wilton-Ely, A. Schier and H. Schmidbaur, Organometallics 2001, 20, 1895-1897; d) F. Bentiss, M. Traisel and M. Lagrenee, J. Appl. Electrochem. 2001, 31, 41-48; e) F. Bentiss, M. Lebrini, H. Vezin and M. Lagrenee, Mater. Chem. Phys. 2004, 87, 18-23; f) B. Sybo, P. Bradley. A. Grubb, S. Miller, K. J. W. Proctor, L. Clowes, M. R. Lawrie, P. Sampson, A. J. Seed. J. Mat. Chem. 2007, 17, 3406-3410.

[5] A. Shafiee, I. Lalezari, S. Yazdany, A. Pournorouz, J. Pharm. Sci. 1973, 62, 839-842.

[6] I. V. Cohen, J. Heterocycl. Chem. 1979, 16, 806-807.

[7] R. V. Kendall, R. A. Olofson, J. Org. Chem. 1970, 35, 806-808.

[8] R. Stolle, L. Gutmann, J. Prakt. Chem. 1904, 69, 509.

[9] E. Bulka, D. Ehlers, J. Prakt. Chem. 1973, 315, 155-163.

[10] I. Lalezari, A. Shafiee, J. Heterocycl. Chem. 1971, 8, 835-837.

[11] a) M. Jesberger, T. P. Davis, L. Barner, Chem. Rev. 2005, 105, 1387-1391; b) S. Knapp, E. Darout, Org. Lett. 2005, 7, 203-206.

[12] a) I. P. Gray, P. Bhattacharyya, A. M. Z. Slawin, J. D. Woollins, Chem. Eur, J. 2005, 11, 62216227; b) W. Shi, M. S. Fallah, C. E. Anson, A. Rothenberger, Dalton Trans. 2006, 2979-2983; c) W. Shi, M. S. Fallah, L. Zhang, C. E. Anson, E. Matern, A. Rothenberger, Chem. Eur. J. 2007, 13, 598-603.

[13] G. Hua, J. D. Woollins, Angew. Chem., Int. Ed. 2008, DOI:10.1002/anie.200800572.

[14] a) S. Cesarini, N. Colombo, M. Pulici, E. R. Felder, W. K. D. Brill, Tetrahedron 2006, 62, 10223 10226; b) S. Xun, G. LeClair, J. Zhang, X. Chen, J. P. Gao, Z. Y. Wang, Org. Lett. 2006, 8, $1697-$ 1700; c) B. Gierczyk, M. Zalas, Org. Prep. Proced. Int. 2005, 37, 213-216; d) C. T. Brain, J. M. Paul, Y. Loong, P. J. Oakley, Tetrahedron Lett. 1999, 40, 3275-3278; e) H. M. Huang, H. T. Yu, P. L. Chen, J. M. Han, B. Ji, Youji Huaxue 2004, 24, 502-504; f) M. A. Herrero, J. Wannberg, M. 
Larhed, Synlett 2004, 2335-2338.

[15] H. N. Dogan, A. Duran, S. Rollas, G. Sener, M. K. Uysalb, D. Gulenc, Bioorg. Med. Chem. 2002, 10, 2893-2897.

[16] M. Kritsanida, A. Mouroutsou, P. Marakos, N. Pouli, S. Papakonstantinou-Garoufalias, C. Pannecouque, M. Witvrouw, E. deClercq, Il Farmaco 2002, 57, 253-257.

[17] S. Schenone, O. Bruno, A. Ranise, F. Bondavalli, W. Filippelli, G. Falcone, L. Giordano, M. R. Vitelli, Bioorg. Med. Chem. 2001, 9, 2149-2153.

[18] J. Y. Chou, X. Y. Lai, S. L. Pan, G. M. Jow, J. W. Chern, J. H. Guh, Biochem. Pharm. 2003, 66, 115-124.

[19] E. Palaska, G. Sahin, P. Kelicen, N. T. Durlu, G. Altinok, Il Farmaco 2002, 57, 101-107.

[20] Y. Gao, Z. Zhang, Q. Xue, Mat. Res. Bull. 1999, 34, 1867-1874.

[21] a) P. Bhattacharyya, A. M. Z. Slawin, J. D. Woollins, Chem. Eur. J. 2002, 8, 2705-2711; b) P. Bhattacharyya, A. M. Z. Slawin, J. D. Woollins, Angew. Chem., Int. Ed. 2000, 39, 19731975.

[22] Data for 2g: $\mathrm{C}_{14} \mathrm{H}_{9} \mathrm{BrN}_{2} \mathrm{Se}$; FW 364.10; Space group P21; $a=5.7482(8), b=7.1175(12), c=$ 15.549(3) $\AA ; \alpha=90, \beta=98.610(8), \lambda=90^{\circ} ; U=629.00(17) \AA^{3} ; Z=2 ; \mu=6.14 \mathrm{~mm}^{-1}$; Reflections collected $=4310 ;$ Independent reflections $=2286 ; \mathrm{R}_{\mathrm{int}}=0.104 ;$ Final $R$ indices $[\mathrm{I}>2 \sigma(I)] R 1=$ $0.085, \mathrm{w} R 2=0.271$.

[23] G. Hua, Y. Li, A. M. Z. Slawin, J. D. Woollins, Unpublished work.

[24] a) M. A. Beswick, C. N. Harmer, P. R. Raithby, A. Steiner, M. Tombul, D. S. Wright, J. Organomet. Chem. 1999, 574, 267-275; b) H. Hope, C. Knobler, J. D. McCullough, Acta Crystallogr. 1970, B26, 628-640. 


\section{Entry for the Table of Contents}

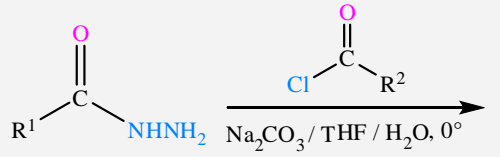

An efficient two-step method for the disubstituted-1,3,4-selenadiazoles by selenating with Woollins' reagent is is reported. Six examples, representing the first preparation of a series of novel 2,5-
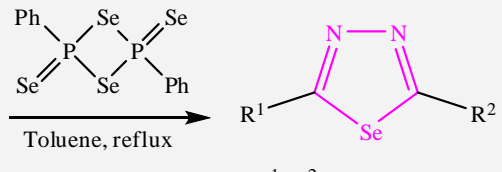

$\mathrm{R}^{1}, \mathrm{R}^{2}=$ Aryl or Alkyl

examples of this class have been characterised crystallographically.
Organoselenium chemistry

Guoxiong Hua, Yang Li, Amy L. Fuller, Alexandra M. Z. Slawin and J.

Derek Woollins*........

Facile Synthesis and Structure of Novel 2,5-Disubstituted 1,3,4Selenadiazoles

Key words: Woollins' reagent / 1,2diacylhydrazines / 2,5-disubstituted1,3,4-selenadiazoles / X-ray strucutre 